Rochester Institute of Technology RIT Scholar Works

Articles

$12-1-2005$

\title{
The ACS Virgo Cluster Survey. X. Half-Light Radii of Globular Clusters in Early-Type Galaxies: Environmental Dependencies and a Standard Ruler for Distance Estimation
}

Andrés Jordán

European Southern Observatory

Patrick Côté

Herzberg Institute of Astrophysics

John P. Blakeslee

The Johns Hopkins University

David Merritt

Rochester Institute of Technology

et al.

Follow this and additional works at: http://scholarworks.rit.edu/article

Recommended Citation

Andrés Jordán et al 2005 ApJ 6341002 https://doi.org/10.1086/497092

This Article is brought to you for free and open access by RIT Scholar Works. It has been accepted for inclusion in Articles by an authorized administrator of RIT Scholar Works. For more information, please contact ritscholarworks@rit.edu. 
ACCEPTED FOR PUblication IN ApJ

Preprint typeset using $\mathrm{LAT}_{\mathrm{E} X} \mathrm{X}$ style emulateapj v. 6/22/04

\title{
THE ACS VIRGO CLUSTER SURVEY X. HALF-LIGHT RADII OF GLOBULAR CLUSTERS IN EARLY-TYPE GALAXIES: ENVIRONMENTAL DEPENDENCIES AND A STANDARD RULER FOR DISTANCE ESTIMATION $^{1}$
}

Andrés Jordán ${ }^{2,3}$, Patrick Côtét ${ }^{4,5}$, John P. Blakeslee ${ }^{6}$, Laura Ferrarese ${ }^{4,5}$, Dean E. Mclaughlin ${ }^{7}$, Simona Mei ${ }^{6}$, Eric W. Penc ${ }^{4}$, John L. Tonry ${ }^{8}$, David Merritt ${ }^{9}$, Miloš Milosavljević ${ }^{10}$, Craig L. Sarazin ${ }^{11}$, Gregory R. SivaKOFF $^{11}$ AND Michael J. West ${ }^{12}$

Accepted for publication in ApJ

\begin{abstract}
We have measured half-light radii, $r_{h}$, for thousands of globular clusters (GCs) belonging to the one hundred early-type galaxies observed in the ACS Virgo Cluster Survey and the elliptical galaxy NGC 4697. An analysis of the dependencies of the measured half-light radii on both the properties of the GCs themselves and their host galaxies reveals that, in analogy with GCs in the Galaxy but in a milder fashion, the average half-light radius increases with increasing galactocentric distance or, alternatively, with decreasing galaxy surface brightness. For the first time, we find that the average half-light radius decreases with the host galaxy color. We also show that there is no evidence for a variation of $r_{h}$ with the luminosity of the GCs. Finally, we find in agreement with previous observations that the average $r_{h}$ depends on the color of GCs, with red GCs being $\sim 17 \%$ smaller than their blue counterparts. We show that this difference is probably a consequence of an intrinsic mechanism, rather than projection effects, and that it is in good agreement with the mechanism proposed in Jordán (2004). We discuss these findings in light of two simple pictures for the origin of the $r_{h}$ of GCs and show that both lead to a behavior in rough agreement with the observations. After accounting for the dependencies on galaxy color, galactocentric radius and underlying surface brightness, we show that the average GC half-light radii $\left\langle r_{h}\right\rangle$ can be successfully used as a standard ruler for distance estimation. We outline the methodology, provide a calibration for its use, and discuss the prospects for this distance estimator with future observing facilities. We find $\left\langle r_{h}\right\rangle=2.7 \pm 0.35 \mathrm{pc}$ for GCs with $(g-z)=1.2 \mathrm{mag}$ in a galaxy with color $(g-z)_{\mathrm{gal}}=1.5 \mathrm{mag}$ and at an underlying surface $z$-band brightness of $\mu_{z}=21 \mathrm{mag} \operatorname{arcsec}^{-2}$. Using this technique, we place an upper limit of $3.4 \mathrm{Mpc}$ on the $1-\sigma$ line-of-sight depth of the Virgo Cluster. Finally, we examine the form of the $r_{h}$ distribution for our sample galaxies and provide an analytic expression which successfully describes this distribution.
\end{abstract}

Subject headings: galaxies: elliptical and lenticular, cD — galaxies: star clusters — globular clusters: general

\section{INTRODUCTION}

The half-light radius, $r_{h}$, represents one of the most fundamental structural properties of globular clusters (GCs). Numerical simulations have shown this quantity

\footnotetext{
${ }^{1}$ Based on observations with the NASA/ESA Hubble Space Telescope obtained at the Space Telescope Science Institute, which is operated by the Association of Universities for Research in Astronomy, Inc., under NASA contract NAS 5-26555

2 European Southern Observatory, Karl-Schwarzschild-Str. 2, 85748 Garching bei München, Germany; ajordan@eso.org

3 Astrophysics, Denys Wilkinson Building, University of Oxford, 1 Keble Road, OX1 3RH, UK

4 Herzberg Institute of Astrophysics, 5071 W. Saanich Road, Victoria, BC V9E 2E7, Canada

${ }^{5}$ Department of Physics \& Astronomy, Rutgers University, Piscataway, NJ 08854, USA

6 Department of Physics and Astronomy, Johns Hopkins University, Baltimore, MD 21218, USA

7 Department of Physics \& Astronomy, University of Leicester, Leicester, LE1 7RH, UK

8 Institute of Astronomy, University of Hawaii, 2680 Woodlawn Drive, Honolulu, HI 96822, USA

9 Department of Physics, Rochester Institute of Technology, 84 Lomb Memorial Drive, Rochester, NY 14623, USA

10 Theoretical Astrophysics, California Institute of Technology, Pasadena, CA 91125, USA

11 Department of Astronomy, University of Virginia, P.O. Box 3818, Charlottesville, VA 22903-0818, USA

12 Department of Physics \& Astronomy, University of Hawaii, Hilo, HI 96720, USA
}

to be fairly constant throughout the dynamical evolution these stellar systems (Spitzer \& Thuan 1972; Lightman \& Shapiro 1978; Murphy, Cohn \& Hut 1990; Aarseth \& Heggie 1998). Moreover, there are reasons to believe that the present day half-light radii are faithful records of the characteristic sizes of the proto-GC clouds; thus, measurements of half-light radii provide unique constraints on the properties of the GCs at the time of their formation (Murray \& Lin 1992).

Until recently, measurements of GC half-light radii were confined to Local Group galaxies where the GCs could be resolved in ground-based images. In the Milky Way, it has long been known that $r_{h}$ increases systematically with galactocentric distance, $R_{g c}$ (van den Bergh 1956). van den Bergh, Morbey \& Pazder (1991) found a scaling relation of the form $r_{h} \propto R_{g c}^{0.5}$ based on an analysis of 98 Galactic GCs. This situation changed dramatically with the launch of the Hubble Space Telescope (HST) which can partially resolve the spatial profiles of GCs well beyond the Local Group; indeed, HST opened the study of GC sizes in external galaxies out to distances of 30 Mpc (e.g., Kundu et al. 1999; Puzia et al. 1999; Larsen et al. 2001). An interesting result to emerge from these studies is the realization that the half-light radii of metal-rich GCs in luminous early-type galaxies appear to be $\sim 20 \%$ smaller than those of their metal-poor counter- 
parts - a finding that has alternatively been interpreted as the result of projection effects (Larsen \& Brodie 2003) or the combined effects of mass-segregation and the dependence of stellar lifetimes on metallicity under the assumption that their half-mass radius is constant (Jordán 2004). Unfortunately, the fields of view have been typically too small to explore possible dependencies of $r_{h}$ on projected $R_{g c}$; only in one galaxy (NGC 4365) has a clear increase of $r_{h}$ with projected $R_{g c}$ been found in the outermost regions (Larsen et al. 2001).

The prospects for using the sizes of GCs for distance estimation dates back at least to the work of Shapley \& Sawyer (1927), who state that "measures of diameter [of GCs] are chiefly useful in the determination of relative distances". Recently, Kundu \& Whitmore (2001) used archival $H S T$ /WFPC2 observations for a sample of 28 galaxies to argue that the median half-light radii for GCs in early-type galaxies is constant at $\left\langle r_{h}\right\rangle=2.36 \pm 0.4 \mathrm{pc}$. This led them to suggest that $\left\langle r_{h}\right\rangle$ could be used as a distance indicator, although they noted that their sample showed a tendency for the physical size of GCs to increase with galaxy distance, a trend they attributed to instrumental effects.

In this paper, we use the GCs from our large and homogeneous survey of early-type galaxies in the Virgo Cluster to explore the dependencies of half-light radii on various properties of the GCs and their host galaxies. We describe the observations and data reductions in $\S 2$. An exploration of the dependencies of half-light radii on the properties of the GCs and their host galaxies is given in $\S 3$. Theoretical implications of the measured half-light radii are briefly discussed in $\S 4$. After accounting for various environmental dependencies, we show in $\S 5$ that $\left\langle r_{h}\right\rangle$ can be successfully used as a distance indicator and we provide a method and calibration for its use. In $\S 6$, we study the form of the observed $r_{h}$ distribution in earlytype galaxies and present an analytic expression which successfully describes this distribution across our sample. We summarize and conclude in $\S 7$. An appendix gives a description of the algorithm used to measure structural parameters, including half-light radii, for GCs in the ACS Virgo Cluster Survey.

\section{OBSERVATIONS}

A sample of 100 early-type galaxies in the Virgo cluster was observed as part of the ACS Virgo Cluster Survey (ACSVCS; Côté et al. 2004). Each galaxy was observed in the F475W $(\simeq$ Sloan $g$ ) and F850LP ( $\simeq$ Sloan $z$ ) bandpasses for a total of $720 \mathrm{~s}$ and $1210 \mathrm{~s}$ respectively. Additionally, the X-ray faint early-type galaxy NGC 4697 was observed with an identical strategy as part of a joint Chandra-HST program (GO-10003; PI = C.L. Sarazin). Data reductions were performed as described in Jordán et al. (2004a). For all GC candidates, a half-light radius is measured by fitting PSF-convolved King (1966) models to the observed light distribution. The methodology used to measure $r_{h}$ is described in Appendix A. Profile fitting is carried out independently in both the F475W and F850LP filters. For this work, we estimate $r_{h}$ by averaging the results measured in the two bandpasses. Having two independent measurements in different bands lets us estimate systematic uncertainties arising from the adopted PSF models. We find that the measurements in the two bands have systematic differences of $\sim 0.05 \mathrm{WFC}$ pixels, or $\sim 0.2 \mathrm{pc}$ at the distance of Virgo (Mei et al. $2005 \mathrm{~b})$. We have thus added in quadrature a systematic error of 0.05 pixels, or 0 '. 0025 , to the measured $r_{h}$ values.

The construction of a clean and complete GC catalog is an essential but difficult part of all studies of extragalactic GC systems. While the full details of the GC selection process will be described elsewhere, along with the presentation of the GC catalogs (Jordán et al., in preparation), we briefly outline the technique here. Making use of the measured $r_{h}$ and $z$-band magnitude and catalogs of the expected contamination taken from blank control fields and constructed for each galaxy individually, we select GCs via maximum likelihood estimation of a mixture-model of GCs and contaminants. Due to the difficulty of distinguishing extended GCs from background galaxies, sources with $r_{h}>0$ '! $128(\sim 10 \mathrm{pc}$ at the mean Virgo distance) are eliminated as GC candidates before estimating the mixture model. This method assigns to each candidate a probability, $p_{\mathrm{gc}}$, that it is a GC. In this work, we consider only those sources for which $p_{\mathrm{gc}} \geq 0.5$.

When analysing the $r_{h}$ measurements, it is crucial to minimize or avoid any selection and contamination effects. In order to create a clean sample for structural parameters studies, we have restricted our sample to GC candidates to those with

$$
\begin{aligned}
z & \leq 22.9 \mathrm{mag} \\
0.6 \leq(g-z) & \leq 1.7 \mathrm{mag}
\end{aligned}
$$

Note that the selection on magnitude corresponds roughly to the expected turnover of the GC luminosity function at the distance of Virgo. Additionally, we have imposed on each program galaxy a selection on projected galactocentric distance. An inner cut, determined with the help of our artificial objects test, ensures that the underlying surface brightness of the galaxy is such that our completeness for objects with $r_{h}=0$ "' 128 is greater than $90 \%$. An outer cut, determined using our control fields, ensures that the expected contamination in our GC samples from background galaxies is at most $5 \%$ of the objects in the sample. The average expected contamination for the samples is $\sim 3 \%$, with the values increasing with decreasing luminosity: i.e., from $\sim 1 \%$ for the giants to $\sim 5 \%$ for the least luminous dwarfs. For our analysis, we considered only those galaxies whose final GC samples contained five or more objects. ${ }^{13}$ This left a final sample of 67 galaxies including NGC 4697. The typical random uncertainties for the measured half-light radii are $\approx 0$.' $003 \approx 0.24 \mathrm{pc}$.

As part of the ACS Virgo Cluster Survey, we have measured distances for most of our target galaxies using the method of surface brightness fluctuations (SBF; Tonry \& Schneider 1988). The reduction procedures for SBF measurements, feasibility simulations for our observing configuration, and the calibration of the $z$-band SBF method have been presented in Mei et al. (2005ab). The complete catalog of SBF distances will be presented in Mei et al. (2005c). With distances to most our targets in hand, we may convert the observed half-light radii from angular into physical units, and thereby examine directly

\footnotetext{
13 Additionally, four faint galaxies located close to luminous giants were eliminated since their own GC systems are overwhelmed by those of their companions. These galaxies are VCC 1297, VCC 1199, VCC 1192 and VCC 1327.
} 
the physical sizes of the GCs. In what follows, we consider only those GCs which belong to the galaxies with measured SBF distances. Our final sample is summarized in Table 1 which lists the 67 galaxies upon which our analysis is based, the blue magnitude, $B_{g}$, from the Virgo Cluster Catalog of Binggeli, Sandage \& Tammann (1985), the number of GCs satisfying the above selection criteria, the mean GC half-light radius in arcseconds and its uncertainty (not including systematic uncertainties) and alternative names for our sample galaxies.

\section{ENVIRONMENTAL DEPENDENCIES}

As mentioned in $\S 1, \mathrm{GC}$ half-light radii are believed to reflect the physical conditions of the proto-GC clouds at the time of their formation. Thus, any systematic variations in half-light radius can provide potentially powerful insights into the mechanism(s) of GC and galaxy formation.

The various factors that can, in principle, affect the half-light radii of GCs can be naturally divided into factors that change $r_{h}$ individually in each GC, factors that affect the GC system within a galaxy, and factors that change it across galaxies. In what follows, we shall refer to these factors as internal, local and global factors. The variables we will use to parameterize internal effects are the GC colors, $(g-z)_{\mathrm{gc}}$, and $z$-band magnitudes. Local factors will be parameterized using the variables $\log \left(r / r_{e}\right)$, where $r_{e}$ is the effective radius obtained from our isophotal analysis (Ferrarese et al. 2005, in preparation), and the underlying $z$-band surface brightness of the galaxy, $\mu_{z}$, at the position of each GC. Note that $\mu_{z}$ is preferred over its $g$-band counterpart because it is a better tracer of the underlying stellar mass and is less sensitive to dust obscuration. Finally, global parameters are taken to be the absolute blue magnitude of the host galaxy, $M_{B}$, and its corresponding color, $(g-z)_{\text {gal }}$ (Binggeli, Sandage \& Tammann 1985; Côté et al. 2004; Ferrarese et al. 2005). ${ }^{14}$ We use our SBF distance moduli (Mei et al. 2005bc) to calculate absolute magnitudes on a galaxy-by-galaxy basis.

Many of these properties are highly correlated, so the extraction of the intrinsic dependence on each of them is not entirely straightforward. For example, not only are $M_{B}$ and $(g-z)_{\text {gal }}$ correlated, but both quantities are themselves correlated with the mean color of the GC systems and the fraction of GCs which belong to the metal-rich component (with brighter and redder systems having a higher fraction of metal-rich GCs; e.g., Peng et al. 2005). Moreover, the dependence of the average half-light radius, $\left\langle r_{h}\right\rangle$, on $(g-z)_{\mathrm{gc}}$ (see below) will affect the inferred dependence of $\left\langle r_{h}\right\rangle$ on $\mu_{z}$ through differing proportions of metal-rich and metal-poor GCs as a function of radius: i.e., red and blue GCs are often found to have different spatial distributions, with the latter having a more extended spatial distribution (Kissler-Patig et al. 1997; Lee, Kim \& Geisler 1998; Côté et al. 2001; Dirsch et al. 2003). ${ }^{15}$ Likewise, the dependence of $\left\langle r_{h}\right\rangle$

\footnotetext{
14 For NGC 4697, which is not a member of the Virgo Cluster and hence does not appear in the catalog of Binggeli, Sandage \& Tammann (1985), we use the integrated blue magnitude from NED: $B=10.14$ mag.

15 While we will refer throughout this work to $\left\langle r_{h}\right\rangle$ as the average or median of $r_{h}$, we actually calculate its value using the biweight location estimator (Beers et al. 1990).
}

TABLE 1

Mean Half-Light Radi for ACS Virgo Cluster Survey GALAXIES AND NGC 4697

\begin{tabular}{|c|c|c|c|c|}
\hline $\mathrm{VCC}$ & $\begin{array}{c}B_{g} \\
(\mathrm{mag})\end{array}$ & $N_{\mathrm{gc}}$ & $\begin{array}{c}\left\langle r_{h}\right\rangle \\
\left({ }^{\prime \prime}\right)\end{array}$ & Other Names \\
\hline 1226 & 9.31 & 392 & $0.033 \pm 0.001$ & N4472, M49 \\
\hline 1316 & 9.58 & 907 & $0.032 \pm 0.000$ & N4486, M87 \\
\hline 1978 & 9.81 & 398 & $0.029 \pm 0.000$ & N4649, M60 \\
\hline 881 & 10.06 & 199 & $0.036 \pm 0.001$ & N4406, M86 \\
\hline 798 & 10.09 & 211 & $0.035 \pm 0.001$ & N4382, M85 \\
\hline 763 & 10.26 & 257 & $0.030 \pm 0.001$ & N4374, M84 \\
\hline 731 & 10.51 & 333 & $0.024 \pm 0.000$ & N4365 \\
\hline 1903 & 10.76 & 190 & $0.033 \pm 0.001$ & N4621, M59 \\
\hline 1632 & 10.78 & 244 & $0.031 \pm 0.001$ & N4552, M89 \\
\hline 1231 & 11.10 & 136 & $0.034 \pm 0.001$ & N4473 \\
\hline 1154 & 11.37 & 93 & $0.031 \pm 0.001$ & N4459 \\
\hline 1062 & 11.40 & 98 & $0.038 \pm 0.001$ & $\mathrm{~N} 4442$ \\
\hline 2092 & 11.51 & 41 & $0.037 \pm 0.002$ & N4754 \\
\hline 759 & 11.80 & 86 & $0.036 \pm 0.001$ & $\mathrm{~N} 4371$ \\
\hline 369 & 11.80 & 115 & $0.037 \pm 0.001$ & $\mathrm{~N} 4267$ \\
\hline 1692 & 11.82 & 56 & $0.038 \pm 0.002$ & N4570 \\
\hline 2000 & 11.94 & 116 & $0.037 \pm 0.001$ & N4660 \\
\hline 1664 & 12.02 & 79 & $0.036 \pm 0.001$ & N4564 \\
\hline 944 & 12.08 & 44 & $0.033 \pm 0.001$ & N4417 \\
\hline 1938 & 12.11 & 51 & $0.041 \pm 0.002$ & N4638 \\
\hline 1279 & 12.15 & 74 & $0.039 \pm 0.002$ & $\mathrm{~N} 4478$ \\
\hline 355 & 12.41 & 8 & $0.036 \pm 0.003$ & N4262 \\
\hline 1619 & 12.50 & 17 & $0.037 \pm 0.002$ & N4550 \\
\hline 1883 & 12.57 & 32 & $0.039 \pm 0.002$ & N4612 \\
\hline 1242 & 12.60 & 63 & $0.036 \pm 0.001$ & $\mathrm{~N} 4474$ \\
\hline 784 & 12.67 & 15 & $0.033 \pm 0.001$ & $\mathrm{~N} 4379$ \\
\hline 1537 & 12.70 & 8 & $0.032 \pm 0.003$ & $\mathrm{~N} 4528$ \\
\hline 778 & 12.72 & 25 & $0.030 \pm 0.002$ & N4377 \\
\hline 828 & 12.84 & 16 & $0.032 \pm 0.002$ & $\mathrm{~N} 4387$ \\
\hline 1321 & 12.84 & 14 & $0.034 \pm 0.002$ & N4489 \\
\hline 1630 & 12.91 & 16 & $0.028 \pm 0.002$ & N4551 \\
\hline 1250 & 12.91 & 8 & $0.041 \pm 0.007$ & $\mathrm{~N} 4476$ \\
\hline 1146 & 12.93 & 36 & $0.038 \pm 0.002$ & $\mathrm{~N} 4458$ \\
\hline 1303 & 13.10 & 21 & $0.033 \pm 0.001$ & N4483 \\
\hline 1913 & 13.22 & 31 & $0.043 \pm 0.002$ & N4623 \\
\hline 1475 & 13.36 & 38 & $0.038 \pm 0.003$ & $\mathrm{~N} 4515$ \\
\hline 1178 & 13.37 & 51 & $0.040 \pm 0.002$ & N4464 \\
\hline 1283 & 13.45 & 22 & $0.032 \pm 0.002$ & $\mathrm{~N} 4479$ \\
\hline 1261 & 13.56 & 17 & $0.034 \pm 0.002$ & N4482 \\
\hline 698 & 13.60 & 56 & $0.039 \pm 0.002$ & N4352 \\
\hline 1422 & 13.64 & 21 & $0.044 \pm 0.003$ & I3468 \\
\hline 9 & 13.93 & 9 & $0.043 \pm 0.003$ & I3019 \\
\hline 1910 & 14.17 & 17 & $0.039 \pm 0.003$ & I809 \\
\hline 856 & 14.25 & 21 & $0.046 \pm 0.003$ & I3328 \\
\hline 140 & 14.30 & 7 & $0.038 \pm 0.001$ & I3065 \\
\hline 1087 & 14.31 & 29 & $0.042 \pm 0.002$ & I3381 \\
\hline 1861 & 14.37 & 25 & $0.045 \pm 0.002$ & $\mathrm{I} 3652$ \\
\hline 543 & 14.39 & 10 & $0.043 \pm 0.003$ & U7436 \\
\hline 1431 & 14.51 & 28 & $0.036 \pm 0.001$ & $\mathrm{I} 3470$ \\
\hline 1528 & 14.51 & 24 & $0.039 \pm 0.002$ & I3501 \\
\hline 437 & 14.54 & 16 & $0.054 \pm 0.006$ & U7399A \\
\hline 2019 & 14.55 & 10 & $0.047 \pm 0.004$ & I3735 \\
\hline 1545 & 14.96 & 19 & $0.043 \pm 0.003$ & I3509 \\
\hline 230 & 15.20 & 11 & $0.039 \pm 0.003$ & I3101 \\
\hline 1828 & 15.33 & 7 & $0.060 \pm 0.005$ & $\mathrm{I} 3635$ \\
\hline 1407 & 15.49 & 11 & $0.044 \pm 0.003$ & I3461 \\
\hline 1539 & 15.68 & 17 & $0.043 \pm 0.002$ & \\
\hline GC4697 & 10.14 & 229 & $0.051 \pm 0.001$ & \\
\hline
\end{tabular}

on $(g-z)_{\mathrm{gc}}$ will also influence the dependence of $\left\langle r_{h}\right\rangle$ on $M_{B}$, as more luminous galaxies have redder GC systems.

Our approach will therefore be to first isolate the various dependencies (i.e., internal, local and global) on environment, and then to "correct" the observed $r_{h}$ so that subsequent correlations are unaffected the preceeding dependencies. We will indicate the successively corrected half-light radii by adding primes (i.e., $\left.r_{h}^{\prime}, r_{h}^{\prime \prime}\right)$ and will 
denote the final corrected $r_{h}$ (in which all environmental dependencies have been accounted for) with a hat: $\hat{r}_{h}$. Our goals in this portion of the analysis are twofold. First, the individual correlations hold potentially important clues to the processes of GC formation and evolution (§区). And second, we will show that - once the various dependencies on GC color, galaxy color and underlying surface brightness are taken into account $-\left\langle\hat{r}_{h}\right\rangle$ is remarkably constant across our sample, and thus appears to be a promising "standard ruler" for distance estimation $(\S 5)$.

\subsection{Internal Factors}

One of the most remarkable properties of GCs is that their size appear to be independent of their mass: i.e., studies of the Galactic GC system show that $r_{h} \propto M_{\mathrm{gc}}^{0}$ (McLaughlin 2000). In fact, this relation is equivalent to one of the defining equations of the "fundamental plane" of GCs, namely, that GC binding energy scales with luminosity as $E_{\mathrm{gc}} \propto L_{\mathrm{gc}}^{2.04}$ (McLaughlin 2000). In Figure 1] we show $r_{h}$ versus $z$-band magnitude for our sample of GCs. A regresssion analysis shows that the linear coefficient in the function $r_{h}=b_{z} z+a_{z}$ is not significant for this sample, meaning that, as expected, GC radii in the ACSVCS are independent of luminosity. We have also performed fits of this same functional form for all galaxies individually. The resulting average linear terms as a function of absolute magnitude are shown in Figure 2 which gives the weighted average slope in each magnitude bin. It is apparent that, across the factor of 350 in luminosity spanned by our sample galaxies, there is no evidence for a variation in the GC size with luminosity. It should be noted that this statement applies only to those clusters brighter than the turnover of the GC luminosity function $(z \leq 22.9)$ due to the construction of the sample. To the extent that the $z$-band luminosity reflects GC mass, this finding provides strong evidence that $r_{h} \propto M_{\mathrm{gc}}^{0}$.

On the other hand, previous HST studies of bright ellipticals have shown that, in the mean, the half-light radii of GCs, $\left\langle r_{h}\right\rangle$, depends on their color $(g-z)_{\mathrm{gc}}$, with the red GCs being $\approx 20 \%$ smaller than their blue counterparts (Kundu \& Whitmore 1998; Kundu et al. 1999; Puzia et al. 1999; Larsen, Forbes \& Brodie 2001; Barmby, Holland \& Huchra 2002; Larsen et al. 2001). As mentioned above, this color dependence will have implications for other $r_{h}$ correlations: in particular, it will affect correlations between $\left\langle r_{h}\right\rangle$ and $M_{B},(g-z)_{\text {gal }}, \log \left(r / r_{e}\right)$ and $\mu_{z}$. To avoid these complications, we choose to temporarily restrict the GC sample to blue clusters only, with $(g-z)_{\mathrm{gc}}<1.05$. Transforming from color to metallicity as in Jordán et al. (2004b), this cut corresponds to a metallicity selection of $[\mathrm{Fe} / \mathrm{H}] \lesssim-1.25$. Once we have characterized the remaining dependencies, we will return to address the issue of the color dependence of GC half-light radii. While requiring the GCs to lie in a restricted range of color will not, in principle, entirely eliminate any dependency, it will certainly reduce it to a very small level. We note that the models presented in Jordán (2004) for the variation of $r_{h}$ with $(g-z)_{\mathrm{gc}}$ which interpret the observed difference in size between red and blue GCs as a joint consequence of mass segregation and depedence of stellar lifetimes on metallicity -

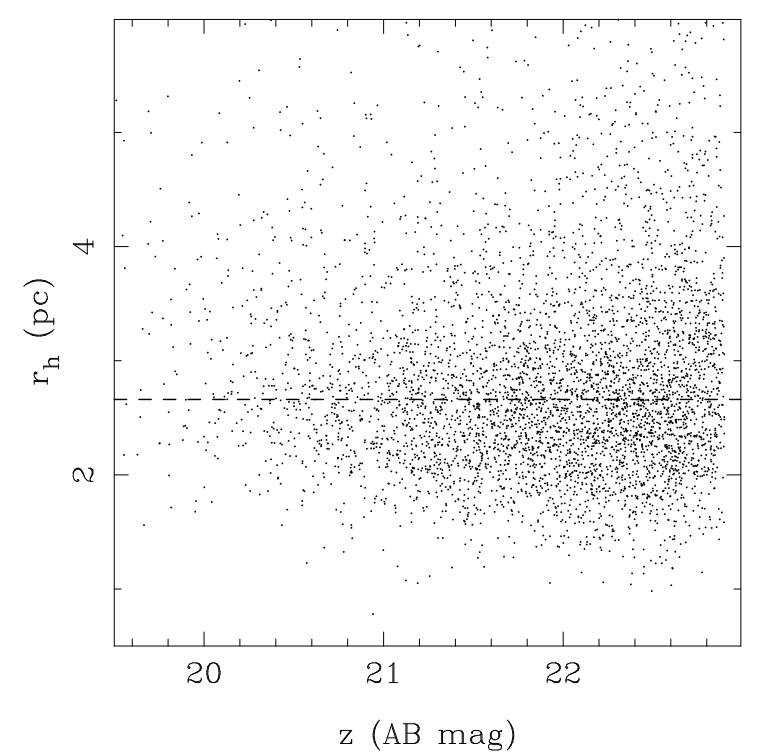

FIG. 1.- Half-light radius, $r_{h}$, plotted as a function of $z$-band magnitude for the combined GCs of our sample galaxies. The data are consistent with $\left\langle r_{h}\right\rangle$ being independent of magnitude. The dashed line marks the median value of $\left\langle r_{h}\right\rangle=2.66 \mathrm{pc}$.

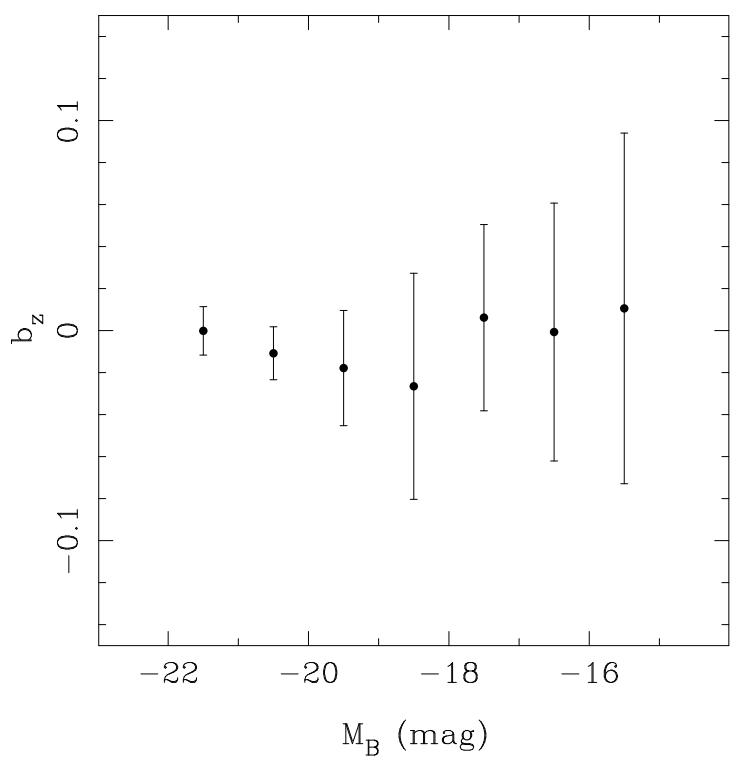

FIG. 2.- Average coefficient $b_{z}$ calculated in one magnitude bins for galaxies in the ACS Virgo Cluster Survey, plotted against their absolute blue magnitude, $M_{B}$. The $b_{z}$ have been obtained from a linear fit of the relation $\log r_{h}=b_{z} z+a$ to the GC data for each galaxy.

predict very little variation for GCs with $[\mathrm{Fe} / \mathrm{H}] \lesssim-1$.

\subsection{Local Factors}

Using our restricted sample of blue GCs, we now search for correlations between the observed $r_{h}$ with projected galactocentric radius, $r_{p}$, and local galaxy surface brightness, $\mu_{z}$. In the two left columns of Figure 3 we show $\log r_{h}$ versus $r_{p}$ in units of the effective radius $r_{e}$ for the eight brightest galaxies in our sample; in the two right columns, we show $\log r_{h}$ versus $\mu_{z}$ for these same eight galaxies. It is clear that the $r_{h}$ increases with both quantities, albeit rather mildly. We have performed linear fits of the form $\log r_{h}=a_{r}+b_{r} \log \left(r / r_{e}\right)$ and 


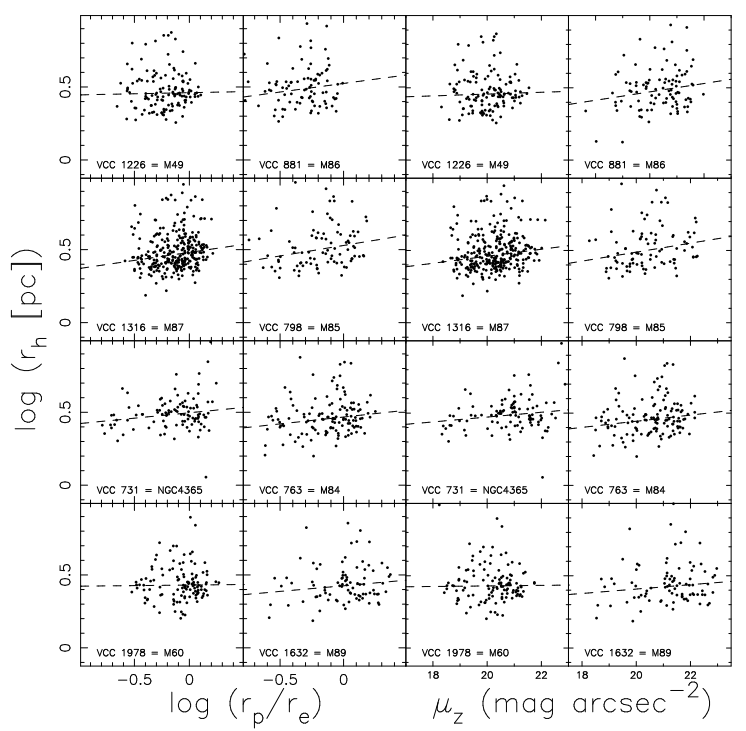

FIG. 3.- (Two Leftmost Columns) Logarithm of GC half-light radius plotted as a function of $\log \left(r_{p} / r_{e}\right)$, where $r_{e}$ is the galaxy effective radius measured from the ACS/WFC images. Only GCs with $(g-z)_{\mathrm{gc}}<1.05$ and belonging to the eight brightest galaxies are shown in this figure. The dashed lines indicate the best fitlinear relations. Galaxy names are indicated in each panel. (Two Rightmost Columns) Logarithm of GC half-light radius plotted as a function of the galaxy surface brightness in the $z$-band, $\mu_{z}$. Only GCs with $(g-z)_{\mathrm{gc}}<1.05$ and beloginging to the eight brightest galaxies are shown in this figure. The dashed lines indicate the best fit-linear relations. Galaxy names are indicated in each panel.

$\log r_{h}=a_{\mu}+b_{\mu} \mu_{z}$ for all galaxies in our sample. We show the resulting values $b_{r}$ and $b_{\mu}$, plotted as a function of galaxy absolute magnitude, $M_{B}$, in Figure 4 We conclude that most galaxies in the sample show evidence for an increase in $r_{h}$ with increasing $r_{p} / r_{e}$ and decreasing surface brightness. A galaxy luminosity term included in the regression analysis is not significant at $99 \%$ level in either case, so we conclude that a single slope sufficiently describes these increases across the entire sample. The weighted average slopes for the fitted relations are $b_{\mu}=0.016 \pm 0.003$ and $b_{r}=0.07 \pm 0.01$, which we will take as the coefficients of a universal relation for earlytype galaxies. ${ }^{16}$

A slope of $b_{r} \approx 0.07$ is much lower than that found for GCs in our Galaxy, $r_{h} \propto R_{\mathrm{gc}}^{0.5}$, where $R_{\mathrm{gc}}$ is the Galactocentric distance. However, these relations are not directly comparable because we observe only the projected galactocentric radii, $r_{p}$, for the GCs in our target galaxies. To compare with the Galactic relation, we have performed 100 random projections of the Galactic GC system using the positional data from Harris (1996), simulating what would have been observed with $H S T$ /ACS if the Milky Way was located at the distance of Virgo, and the GC sample was selected in the same fashion as for the ACSVCS targets. Only GCs with $M_{V}<-7.4$ mag (i.e. only the brighter half) were used in order to reproduce the magnitude cut of our sample. To each

16 If all GCs, rather than just the blue ones, are used in the analysis, the coefficients change only slightly, to $b_{\mu}=0.017$ and $b_{r}=0.09$. The direction of the change is as expected from the combination of smaller $\left\langle r_{h}\right\rangle$ and less extended spatial distribution for redder GCs.
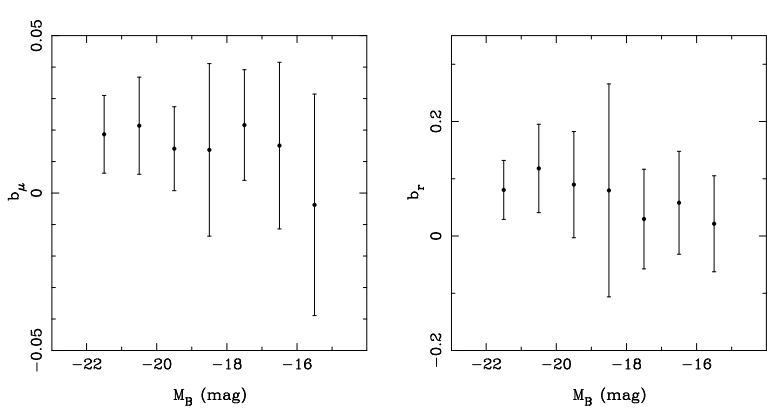

FIG. 4.- (Left) Average coefficient $b_{\mu}$ calculated in one magnitude bins for galaxies in the ACS Virgo Cluster Survey, plotted against their absolute blue magnitude, $M_{B}$. The $b_{z}$ have been obtained from a linear fit of the relation $\log r_{h}=b_{\mu} \mu_{z}+a$ to the GC system of each galaxy. (Right) Average coefficient $b_{r}$ calculated in one magnitude bins for galaxies in the ACS Virgo Cluster Survey, plotted against their absolute blue magnitude, $M_{B}$. The $b_{z}$ have been obtained from a linear fit of the relation $\log r_{h}=b_{r} \log \left(r_{p} / r_{e}\right)+a$ to the GC system of each galaxy.

simulated dataset we have fit a linear relation as above to find $b_{r}$, and recorded the result. These simulations shows that our Galaxy would have been measured to have $b_{r, M W} \sim 0.3$, with a dispersion around this value of $\sim 0.15$. Thus, the slope we find for GCs in early-types in Virgo is significantly shallower than that for GCs in the Galaxy. While we will not explore this issue further in this work, it is an interesting discrepancy that may point to a difference in the formation or dynamical evolution of GC systems in early- versus late-type galaxies, or perhaps, in group versus cluster environments.

While either $\log \left(r_{p} / r_{e}\right)$ and $\mu_{z}$ may be use to parameterize the outward decline in $r_{h}$, it is clear that they are not independent variables: i.e., quite generally, $\mu \propto \log F\left(r_{p} / r_{e}\right)$, where $F$ is an arbitrary continuous function with finite total integral. This does not make the descriptions in terms of the two variables necessarily equivalent but it renders them correlated and forces the choice of one. We choose to work with $\mu_{z}$ as the independent variable since it avoids additional uncertainties that arise due to errors in the determination of $r_{e}$, and in the assumption of spherical symmetry (which is a poor assumption for a number of our program galaxies). Moreover, the use of $\mu_{z}$ accounts implicitly for the non-homologous nature of the galaxy profiles (e.g., Caon, Capaccioli \& D'Onofrio 1993), a behavior which a simple power-law cannot capture.

Using the relation between $r_{h}$ and $\mu_{z}$, we correct the value of $r_{h}$ measured for each $\mathrm{GC}$ to the value expected at an underlying galaxy surface brightness of $\mu_{z}=21 \mathrm{mag} \operatorname{arcsec}^{-2}$. The resulting corrected half-light radius $r_{h}^{\prime}$ is given then by

$$
r_{h}^{\prime} \equiv r_{h} 10^{-0.016\left(\mu_{z}-21\right)} .
$$

Note that the form of the correction tacitly assumes that only the mean of the $r_{h}$ distribution is changing according to the derived relation.

\subsection{Global Factors}

Having examined the internal variation in $r_{h}$, we now turn our attention to global dependencies. In this case, our sample is again restricted in GC color, and the halflight radii have been corrected as given in Equation 2 


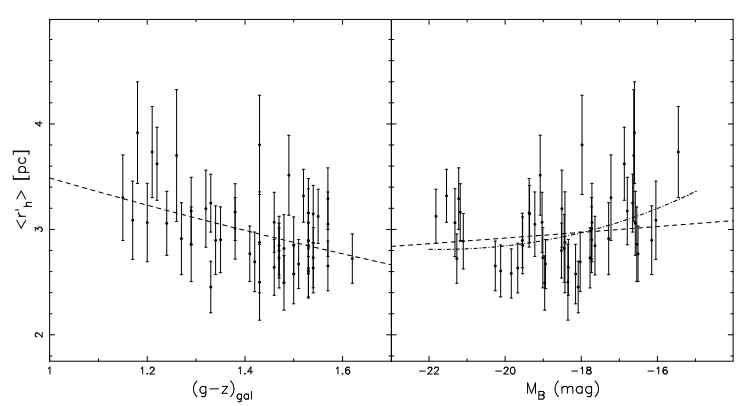

FiG. 5.- (Left) Mean GC half-light radius, $\left\langle r_{h}^{\prime}\right\rangle$, corrected to an underlying surface brightness of $\mu_{z}=21 \mathrm{mag} \operatorname{arcsec}^{-2}$, plotted against galaxy color, $(g-z)_{\text {gal }}$. The dashed line is the least-squares line of best fit, showing a residual dependence of $\left\langle r_{h}^{\prime}\right\rangle$ on $(g-z)_{\text {gal }}$. (Right) $\left\langle r_{h}^{\prime}\right\rangle$ as a function of galaxy absolute blue magnitude, $M_{B}$. The dashed line is the least-squares linear fit to the data. The dotdashed curve is the relation obtained by folding the linear relation seen in the left panel with a quadratic relation between $(g-z)_{\text {gal }}$ and $M_{B}$ inferred from our sample.

i.e., we are working with $r_{h}^{\prime}$ values. Any remaining dependency therefore must arise from global factors. In Figure 5, we show the average half light radius $\left\langle r_{h}^{\prime}\right\rangle$ plotted against galaxy color $(g-z)_{\text {gal }}$ and galaxy blue luminosity $M_{B}$ (left and right panels, respectively). In both cases, there may be residual trends, particularly among the bluer and fainter galaxies, which seem to have somewhat larger $\left\langle r_{h}^{\prime}\right\rangle$. Linear fits of $\log \left\langle r_{h}^{\prime}\right\rangle$ with galaxy color and absolute magnitude give

$$
\log \left\langle r_{h}^{\prime}\right\rangle=(0.459 \pm 0.008)-(0.167 \pm 0.054)\left[(g-z)_{\text {gal }}-1.5\right]
$$

and

$$
\log \left\langle r_{h}^{\prime}\right\rangle=(0.465 \pm 0.010)+(0.004 \pm 0.005)\left(M_{B}+20\right)
$$

The coefficient of $M_{B}$ is not statistically significant, while the color coefficient is significant at a $3 \sigma$ level. Due to the well-known correlation between galaxy color and luminosity (Baum 1959; van den Bergh 1975), the use of either $M_{B}$ or $(g-z)_{\text {gal }}$ should be roughly equivalent as a tracer of the global variation in $r_{h}^{\prime}$. For the ACSVCS sample, we can determine the relationship between these parameters explicitly: Figure [6] shows $(g-z)_{\text {gal }}$ as a function of $M_{B}$, along with the best-fit quadratic relation

$$
(g-z)_{\text {gal }}=-3.2-0.45 M_{B}-0.010 M_{B}^{2},
$$

which is valid in the range $-22 \lesssim M_{B} \lesssim-15$. The need for a quadratic term is a reflection of the fact that the relation flattens out at the high galaxy luminosities, with a plateau in color after a certain luminosity threshold is crossed (cf. Tremonti et al. 2004). This might provide a clue as to why $\log \left\langle r_{h}^{\prime}\right\rangle$ shows little or no dependence on an $M_{B}$ term as compared with $(g-z)_{\text {gal }}$. In any case, due to the better behavior and the added benefit of being independent of distance, we will choose to model this mild dependence using the galaxy color as opposed to its blue luminosity.

We now use the dependence on galaxy color to further correct the half-light radii:

$$
r_{h}^{\prime \prime} \equiv r_{h} 10^{-0.016\left(\mu_{z}-21\right)+0.17\left[(g-z)_{\mathrm{gal}}-1.5\right]} .
$$

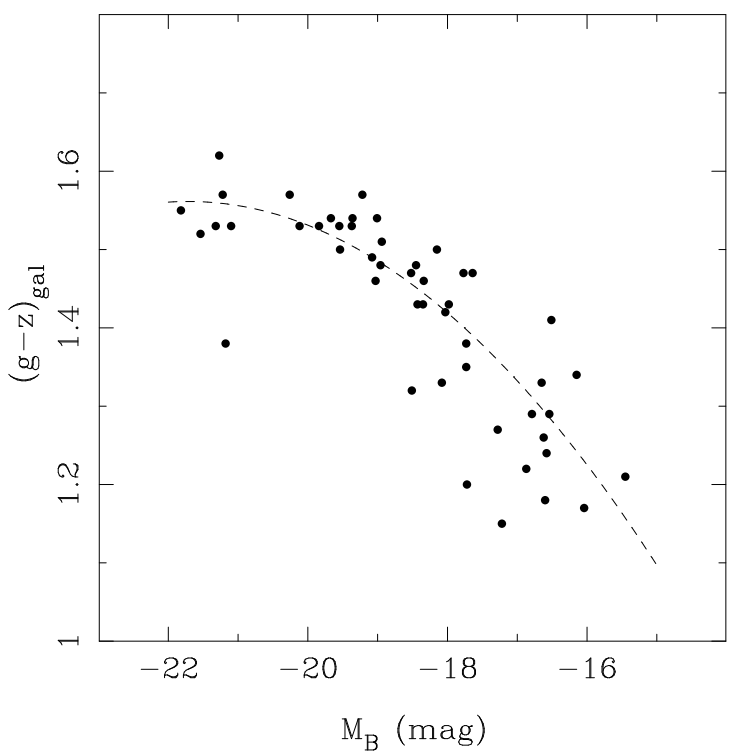

FIG. 6.- Galaxy color, $(g-z)_{\text {gal }}$, versus absolute blue magnitude, $M_{B}$, for galaxies in our sample. The dashed line shows the best-fit quadratic relation in the range $-22 \lesssim M_{B} \lesssim-15$.

\subsection{Dependence on GC Color}

Using the half-light radii corrected according to Equation [6] we now return to the question of the color dependence of half-light radii. In doing so, we drop the restriction to blue GCs imposed in $\S 3.1$

To quantify variations of $r_{h}^{\prime \prime}$ with color, we must have enough GCs in any given color bin to get an accurate measure of the mean behavior. This requirement imposes a limitation on the luminosity of the galaxies to be studied, as less luminous galaxies have GC systems which are almost exclusively metal-poor (i.e., many of the dwarfs have only a handful of GCs with $\left.(g-z)_{\mathrm{gc}} \gtrsim 1.2\right)$. Thus, we have decided to create composite GC sets by combining galaxies in color bins created by demanding at least 100 GCs with colors in the range $1.3 \leq(g-z)_{\text {gc }} \leq 1.5$. In addition, we consider only those galaxies which have a number of GCs in the range $1.3<(g-z)_{\text {gc }}<1.6$ that is at least $10 \%$ of the number found in the range $0.8<(g-z)_{\mathrm{gc}}<1.1$. The motivation for this condition is that adding galaxies which have purely metalpoor GC systems adds no information to the characterization of the $r_{h}^{\prime \prime}$ color dependence; indeed, because of uncertainties in the measured distances, such galaxies would add scatter only in the blue population while contributing few or no red GCs. Figure [7] shows the resulting GC samples, which in some cases consist of a single galaxy. Also shown are the best-fit linear relations, $\log r_{h}^{\prime \prime}=a(g-z)_{\mathrm{gc}}+b$ (transformed to $\left.r_{h}^{\prime \prime}\right)$, the median values of $r_{h}^{\prime \prime}$ in bins of $(g-z)$ along with their $99 \%$ confidence intervals, and a model from Jordán (2004) with central potential ${ }^{17} W_{0}=9$, normalized such that the model at $[\mathrm{Fe} / \mathrm{H}]=-1.5$ coincides with the median $r_{h}^{\prime \prime}$ at

\footnotetext{
17 Jordán (2004) modelled the effects of mass segregation using multimass isotropic Michie-King models (Gunn \& Griffin 1979), which are characterized by their central potential $W_{0}$, which is the gravitational potential at the center of the cluster normalized by a characteristic velocity variance. A value of $W_{0}=9$ is comparable to a value of $c \sim 1.6$ for the concentration of a single-mass isotropic King (1966) model, with the concentration increasing with $W_{0}$.
} 


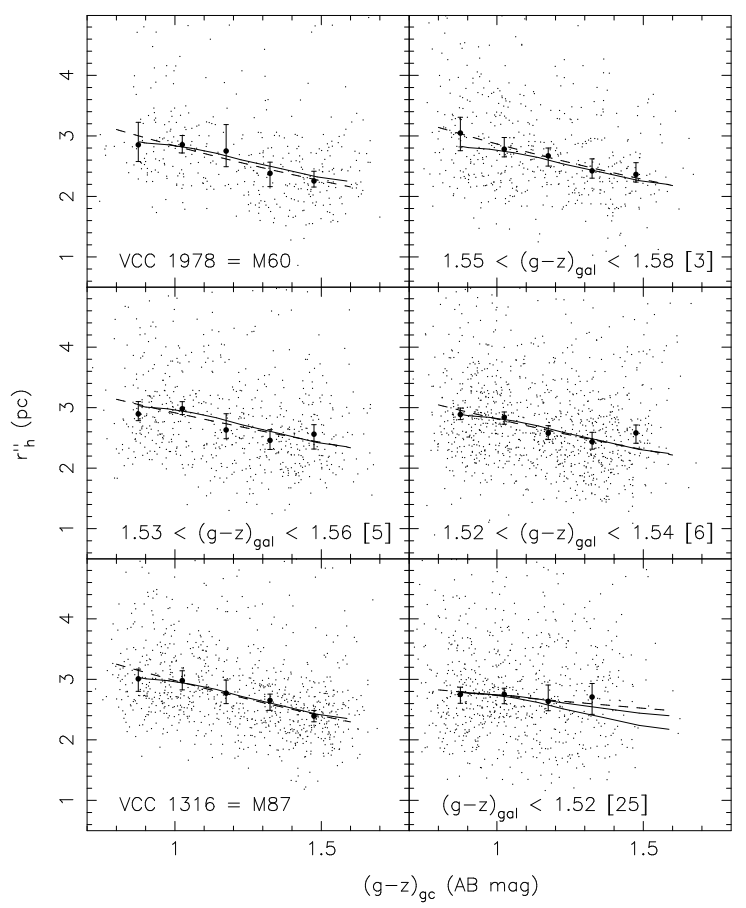

FIG. 7.- GC half-light radii, $r_{h}^{\prime \prime}$, corrected for the effects of surface brightness and galaxy color, plotted against GC color for $\mathrm{GC}$ subsamples formed by combining galaxies in color bins as described in the text. The color bins, or the name of the individual galaxies if only one present, are recorded in the different panels. The number of galaxies used to create the GC subsamples is indicated in brackets. The large symbols indicate the median $r_{h}^{\prime \prime}$ in different GC color bins. The error bars indicate the $99 \%$ confidence intervals. The dashed line represents the best linear fit of the form $\log r_{h}^{\prime \prime} \propto(g-z)_{\mathrm{gc}}$ to the data (transformed to $\left.r_{h}^{\prime \prime}\right)$. The solid line represents the prediction of the behavior of $r_{h}^{\prime \prime}$ with $(g-z)_{\mathrm{gc}}$ from a model from Jordán (2004) with a central potential $W_{0}=9$. For the bin with $(g-z)_{\text {gal }}<1.52$ we have included a second model with $W_{0}=7$ (the upper one).

$(g-z)_{\mathrm{gc}}=1.05$ mag.

It is apparent that the $r_{h}^{\prime \prime}$ show a significant correlation with $(g-z)_{\mathrm{gc}}$ for all of the subsamples in Figure 7 Except for the group of bluer galaxies shown in the bottom left panel, the behavior is very consistent, with

$$
\log r_{h}^{\prime \prime} \propto(-0.17 \pm 0.02)(g-z)_{\mathrm{gc}},
$$

valid in the range $0.8 \lesssim(g-z)_{\mathrm{gc}} \lesssim 1.6$. The bluer bin shows a somewhat shallower behavior, with a slope of $-0.09 \pm 0.04$, but still within $\sim 2 \sigma$ of the mean behavior of the redder galaxies. Because the bluer bin required 25 galaxies for its construction, it is much more prone to uncertainties arising from errors in the measured distances. Futhermore, these galaxies contain few red GCs, so their usefulness in probing the relation between $r_{h}^{\prime \prime}$ and $(g-z)_{\mathrm{gc}}$ is limited. We will therefore assume that the behavior exhibited by galaxies with $(g-z)_{\text {gal }} \gtrsim 1.52$ is valid universally, and will use this relation to perform the final correction of the GC half-light radii (see below).

It is remarkable how well a single model from Jordán (2004), with $W_{0}=9$, does in matching the data in Figure 7 With the possible exception of the bin containing the bluest galaxies, it reproduces the observed trends very well. As mentioned in $\$$ only two mechanisms have been proposed to explain the observed tendency for red
GCs to be smaller than their blue counterparts. Larsen \& Brodie (2003) proposed that this effect could be the consequence of projection effects, combined with the differing spatial distributions of the red and blue GCs. The red GCs are observed to be more centrally concentrated so that, if the GCs follow a relationship between galactocentric distance and $\left\langle r_{h}\right\rangle$ which is similar to that of GCs in the Milky Way, projection effects can lead to the red GCs appearing systematically smaller (due to the fact that they are, on average, physically closer to the galaxy center). As noted by Larsen \& Brodie (2003), however, this mechanism requires some fine-tuning to work. Jordán (2004), on the other hand, has proposed that the observed size difference can be explained as the consequence of mass segregation within individual GCs, combined with the dependence of stellar lifetimes on metallicity. Simply stated, if the half-mass radii are assumed to be independent of metallicity, the half-light radii will, at fixed age, differ because the brightest stars, which dominate the overall light profile, will be more massive in red (metal-rich) GCs and thus more centrally concentrated.

We can use our new measurements to discriminate between these alternatives. First, we note that our observations point to a significantly shallower relation between $r_{h}$ and galactocentric radius in early-type galaxies than the one observed in our Galaxy. This latter relation was assumed by Larsen \& Brodie (2003) and is necessary for the projection mechanism to explain the observed trend. Using shallower relations they found that projection effects are unable to reproduce the observed size difference. Thus, our measurements seem to rule out projection effects as the primary mechanism responsible for the observed difference, although it is certainly possible that they play some part in its origin.

In addition, the importance of projection effects is expected to depend on the fraction of the galaxy surveyed, as they are expected to decrease strongly beyond one effective radius of the GC system, and to disappear entirely as $r_{p} \rightarrow \infty$ (Larsen \& Brodie 2003). The galaxies with $(g-z)_{\text {gal }} \gtrsim 1.52$ span an enormous range in $r_{\text {lim }} / r_{e}$, where $r_{\text {lim }}$ is the upper radii for inclusion of GCs in each galaxy and $r_{e}$ is the effective radius of the galaxy light. These galaxies have $1 \lesssim r_{\lim } / r_{e} \lesssim 20$, with a median of $r_{\lim } / r_{e}=3.3$ and an interquartile range of $\sim 8$. That the observed difference in $r_{h}$ is observed to be nearly identical among these galaxies argues strongly for a mechanism that is independent of projection effects. It might be argued that the fact that the decrease in size seems to be milder for the bluest galaxies is consistent with at least some variation due to projection effects. But the distribution of $r_{\lim } / r_{e}$ for the galaxies in the bluest bin is not extremely different from those in the redder ones, having $2 \lesssim r_{\lim } / r_{e} \lesssim 25$, a median of $r_{\lim } / r_{e}=4.8$ and an interquartile range of 5.5. Thus, it would be fortuitous that the change of regime occurs between samples with median $r_{\lim } / r_{e}$ of $\sim 3.3$ and $\sim 4.8$.

The mechanism of Jordán (2004) seems to fare better, despite the fact that it is a clear oversimplification to compare the data with a single model with $W_{0}=9$. In reality, GCs will span a range in $W_{0}$, and there could well exist a systematic difference in the average central potential of GCs belonging to the blue (low-luminosity) galax- 


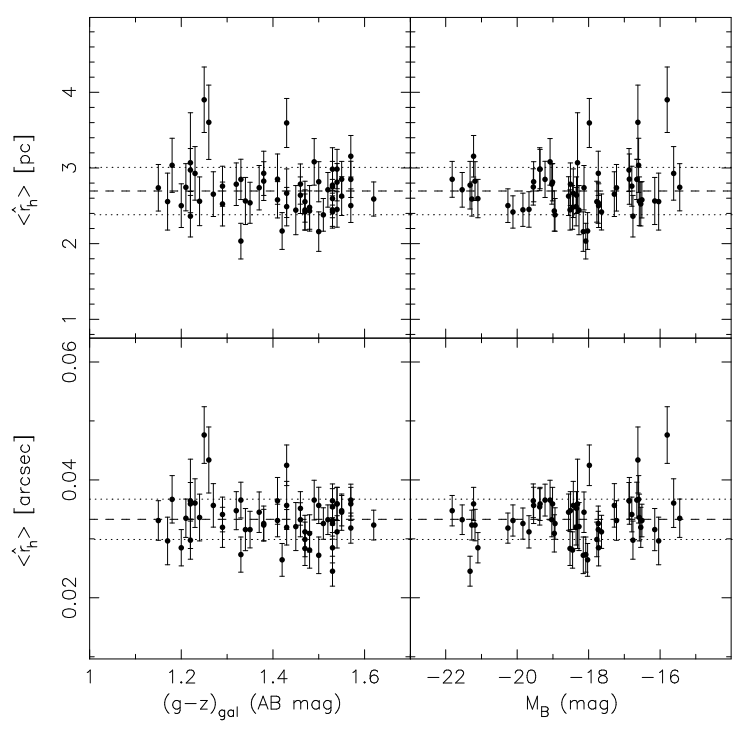

FIG. 8.- (Upper Panels) Fully corrected mean GC half-light radius, $\left\langle\hat{r}_{h}\right\rangle$, in parsecs, plotted as a function of galaxy color, $(g-$ $z)_{\text {gal }}$, in the left panel, and against and absolute blue magnitude, $M_{B}$, in the right panel. The dashed line indicates the biweight estimate of the mean, $\left\langle\hat{r}_{h}\right\rangle=2.72 \mathrm{pc}$. The dotted lines indicate the dispersion of 0.35 pc. (Lower Panels) Fully corrected mean GC half-light radius, $\left\langle\hat{r}_{h}\right\rangle$, in arcseconds, plotted as a function of galaxy color, $(g-z)_{\text {gal }}$, in the left panel, and against and absolute blue magnitude, $M_{B}$, in the right panel. The dashed line indicates the biweight estimate of the mean, $\left\langle\hat{r}_{h}\right\rangle=0{ }^{\prime \prime} 033$. The dotted lines indicate the dispersion of $0{ }^{\prime \prime} 0035$. VCC731, a member of the W Cloud, has been excluded from the computation of these quantities.

ies. As we show in Figure 7 a model with $W_{0}=7$ for the bluer bin is able to reproduce the observations quite well. In any case, the overall conclusion to be drawn from the data in Figure 7 is that, whatever is responsible for the size difference between red and blue GCs does, this difference does not seem to arise mainly from projection effects but is instead the result of some property intrinsic to the GCs.

\subsection{Combined results}

Having systematically explored the variations in halflight radii with a number of internal, local, and global factors, we now use the observed dependencies to correct the individual $r_{h}$ measurements to the expected value for a GC with color $(g-z)_{\mathrm{gc}}=1.2 \mathrm{mag}$, at an underlying surface brightness of $\mu_{z}=21 \mathrm{mag} \operatorname{arecsec}^{-2}$, in a galaxy with $(g-z)_{\text {gal }}=1.5$ mag. In other words, we assume that only the mean of the $r_{h}$ distribution is changing to the above scaling relations, so that the corrected radii, $\hat{r}_{h}$, are given by

$$
\hat{r}_{h} \equiv r_{h} 10^{-0.016\left(\mu_{z}-21\right)+0.17\left[(g-z)_{\text {gal }}-1.5\right]+0.17[(g-z)-1.2]} .
$$

We plot $\left\langle\hat{r}_{h}\right\rangle$ for our sample galaxies, in both arcseconds and parsecs, against $(g-z)_{\mathrm{gc}}$ and $M_{B}$ in Figure 8 It is clear that $\left\langle\hat{r}_{h}\right\rangle$ is remarkably constant across our sample galaxies. As such, this quantity appears to be a "standard ruler", suitable for use as an extragalactic distance indicator. We shall explore this issue in more detail in $\$ 5$

We emphasize that none of the dependencies discussed above are likely to be due to selection effects. Compact clusters at large galactocentric radii (or, equivalently, at low surface brightness) would be readily detected and measured in our survey. The inner radial cut imposed on each galaxy ensures that we are highly complete for extended GCs. The decrease in $\left\langle r_{h}^{\prime}\right\rangle$ with galaxy color could be suspected to be due to contamination preferentially affecting the small GC systems of faint, blue galaxies. However, we have accurate estimates of the expected contamination measured directly from our control fields (Peng et al. 2005, in preparation) so this is unlikely to be the case.

While from an observational point of view, the local and global factors can be separated naturally, they could in principle arise from a single relation which determines $r_{h}$. As a simple illustration, let us assume that $r_{h}$ is related to the surface mass density, $\Sigma_{\text {gal }}$, through the relation $\left\langle r_{h}\right\rangle \propto \Sigma_{\text {gal }}^{-x} \equiv\left[\left(M_{\text {gal }} / L_{\text {gal }}\right) I_{\text {gal }}\right]^{-x}$, where $I_{\text {gal }}$ denotes the galaxy surface brightness and $\left(M_{\text {gal }} / L_{\text {gal }}\right)$ its mass-to-light ratio. Clearly, in this case

$$
\log \left\langle r_{h}\right\rangle \propto-x \log \left(M_{\text {gal }} / L_{\text {gal }}\right)+0.4 x \mu_{z} .
$$

The mass-to-light ratio will vary systematically with both the galaxy luminosity and color. Taking $\left(M_{\text {gal }} / L_{\text {gal }}\right) \propto L_{\text {gal }}^{0.3}$ from Cappellari et al. (2005), we get

$$
\log \left\langle r_{h}\right\rangle \propto 0.12 x M_{B}+0.4 x \mu_{z} .
$$

where we have used the fact that $L_{\text {gal }} \propto 10^{-0.4 M_{B}}$. Under this simple hypothesis, a value of $x \sim 0.04$ would be a reasonable description of the observations (cf. eqs 4 and 8). This simple example serves to show that empirical trends can guide our theoretical understanding of both $\mathrm{GC}$ formation and galactic structure.

We will now explore the implications of the observed global behavior for models which make quantitative predictions regarding the half-light radii of GCs and their dependence on environment.

\section{THEORETICAL CONSIDERATIONS}

Our finding that the half-light radii of GCs show clear correlations with some properties of their host galaxies should not obscure the remarkable fact that over a factor of $\sim 350$ in galaxy luminosity, $\left\langle r_{h}\right\rangle$ varies by only $\sim 10 \%$ (after accounting for internal and local factors). Clearly, one could assume that, to first order, $\left\langle r_{h}\right\rangle$ is constant: $\left\langle r_{h}\right\rangle \sim L_{\text {gal }}^{0}$. Thus, our results demonstrate that any paradigm for the formation of GCs should predict an exponent $\eta$ in the relation $\left\langle r_{h}\right\rangle \sim L_{\text {gal }}^{\eta}$ which is very close to zero. In this section, we show that two possible hypothesis for the origin of the observed $r_{h}$ of GCs do lead to roughly this result. It should be stressed at the onset, however, that the level of theoretical understanding we have on process of GC formation is sketchy at best. Indeed, the observations are far ahead, in the sense that they provide an increasingly clear, albeit sometimes puzzling, set of constraints to which any theory of GC formation must conform.

\subsection{Half-Light Radii as the Result of Pressure-Confined Proto-GC Clouds}

It has been suggested that the half-light radii of GCs reflect the structure of self gravitating proto-GC clouds 
which, at the time of GC formation, were in pressure equilibrium with the surrounding gas. The structure of such clouds has been calculated by McLaughlin \& Pudritz (1996), who determine that their limiting radius satisfies the relation $R_{\lim } \propto M^{0.5} P_{\text {gas }}^{-1 / 4}$ (cf. equation 2.11 in McLaughlin \& Pudritz 1996), where $P_{\text {gas }}$ is the pressure of the ambient gas. If we posit that the average $r_{h}$ observed today is linearly related to $R_{\text {lim }}$, then it follows that $\left\langle r_{h}\right\rangle \propto M^{0.5} P_{\text {gas }}^{-1 / 4}$, where we assume that GCs of differing masses that form at approximately the same radius in a galaxy come from clouds with differing velocity dispersions. It is immediately apparent that this hypothesis is in strong conflict with observations due to the factor $M^{0.5}$. The observational evidence presented in $\S 3$ argues for $\left\langle r_{h}\right\rangle \propto M^{0}$. To resolve this discrepancy one might propose that the star formation efficiency scales as some power of the proto-GC cloud binding energy (e.g., Ashman \& Zepf 2001). While such a scaling relation could, in principle, erase the mass dependence, there is no physical basis to justify such a scaling. In any case, we will carry on by supposing that a mechanism exists to render this pressure support picture viable, and examine what this scenario predicts for the global behavior of $\left\langle r_{h}\right\rangle$ with host galaxy luminosity.

We begin by supposing that $\left\langle r_{h}\right\rangle \propto P_{\text {gas }}^{-1 / 4}$. If the ambient pressure is distributed like the dark matter, then averaging over an entire galaxy gives

$$
\left\langle r_{h}\right\rangle \propto P_{\text {ave }}^{-1 / 4} \propto\left(\frac{M_{\text {vir }}^{2}}{r_{\text {vir }}^{4}}\right)^{-1 / 4}
$$

where $M_{\text {vir }}$ and $r_{\text {vir }}$ are the virial mass and virial radius of the dark halo. We assume now that early-type galaxies all formed at similar large redshifts. The halos should then have the same average density, or $M_{\mathrm{vir}} \propto r_{\mathrm{vir}}^{3}$. Since the circular velocity at the virial radius is given by $v_{\text {vir }}^{2} \equiv$ $G M_{\text {vir }} / r_{\text {vir }}$, we have then that $r_{\text {vir }} \propto v_{\text {vir }}$ and therefore we expect $\left\langle r_{h}\right\rangle \propto v_{\text {vir }}^{-1 / 2}$.

In order to progress further, we assume now that the dark matter halos are described by NFW profiles (Navarro, Frenk \& White 1995). Then the circular velocity $V_{c}$ profile is

$$
\left[\frac{V_{c}(r)}{v_{\mathrm{vir}}}\right]^{2}=\frac{1}{x} \frac{\ln (1+c x)-c x /(1+c x)}{\ln (1+c)-c /(1+c)},
$$

where $x \equiv r / r_{\text {vir }}$ and $c$ is the concentration parameter. This velocity profile implies a peak circular velocity at radius $c x \simeq 2.163$, at which point the value is

$$
\left(\frac{V_{p}}{v_{\text {vir }}}\right)^{2} \simeq 0.2162 \frac{c}{\ln (1+c)-c /(1+c)} \approx 0.2162\left[2.742 c^{0.4}\right]
$$

where the second approximation is accurate to within $\sim 5 \%$ in the range $4 \leq c \leq 20$. Furthermore, simulations show (Bullock et al. 2001) that $c \sim M_{\text {vir }}^{-0.13}$ and thus $V_{p}^{2} \sim c^{0.4} v_{\mathrm{vir}}^{2} \sim v_{\mathrm{vir}}^{1.84}$. Therefore, $\left\langle r_{h}\right\rangle$ is related to the peak circular velocity of the halo through

$$
\left\langle r_{h}\right\rangle \propto v_{\text {vir }}^{-1 / 2} \sim V_{p}^{-0.54}
$$

Finally, Ferrarese (2002) connects $V_{p}$ to the stellar velocity dispersion of the central bulge by

$$
V_{p} \sim \sigma_{0}^{x}, \quad x=0.89 \pm 0.04
$$

and the Faber-Jackson relation has $\sigma_{0} \propto L_{\text {gal }}^{1 / 4}$ so ultimately

$$
\left\langle r_{h}\right\rangle \propto L_{\text {gal }}^{-0.12}
$$

Note that a scaling relation of the form of Equation 16 can also be obtained much more simply by assuming that $\sigma_{0} \propto v_{\text {vir }}$, and as consequence $v_{\text {vir }} \propto L_{\text {gal }}^{1 / 4}$ and $\left\langle r_{h}\right\rangle \propto v_{\text {vir }}^{-1 / 2} \propto L_{\text {gal }}^{-1 / 8}$. In both cases, we get a very mild dependence of $\left\langle r_{h}\right\rangle$ with $L_{\text {gal }}$, albeit stronger than that which is observed. We note that if we had assumed that the average ambient pressure of the proto-GC clouds comes from gas distributed as the present-day starlight and used fundamental plane scaling relations, we would get a similar dependence but of opposite sign. That is to say, in this case we would posit

$$
\left\langle r_{h}\right\rangle \propto P_{\text {ave }}^{-1 / 4} \propto\left(\frac{M_{\text {gal }}^{2}}{r_{e}^{4}}\right)^{-1 / 4}
$$

where $r_{e}$ is the effective radius of the galaxy light. The galactic fundamental plane scalings of $r_{e} \propto L_{\text {gal }}^{0.8}$ and $M / L \propto L_{\text {gal }}^{0.3}$ (Haşegan et al. 2005 and references therein) then give $\left\langle r_{h}\right\rangle \propto L_{\text {gal }}^{0.15}$. Overall, it seems fair to conclude that the pressure hypothesis predicts roughly $\left\langle r_{h}\right\rangle \propto L_{\text {gal }}^{0}$, in accord with the observations from $\S 3$.

\subsection{Half-Light Radii as a Consequence of Tidal Limitation}

An alternative hypothesis is that the half-light radii of GCs are proportional to their tidal radii, $r_{t}$. This runs immediately into the problem of the non-homologous nature of GCs. In fact, the King concentration parameter $c$ is correlated with the GC mass through the rough relation $10^{c} \sim M^{0.4}$, which could be the reflection of an original "fundamental line" (McLaughlin 2000). The $r_{h}$ in a King model is a function of $c$ times $r_{t}$ so that, via the dependence of $c$ on $M$, it follows that a simple proportionality between $r_{h}$ and $r_{t}$ for all GCs cannot be appropriate. Also, there is no clear mechanism to physically transmit the information from the tidal regions to determine the half-light radius. Finally, if the $r_{t}$ are determined by the tidal field of the host galaxy, then we would have $\left\langle r_{h}\right\rangle \propto\left\langle r_{t}\right\rangle \propto M^{1 / 3}$ (Binney \& Tremaine 1987). Thus, we would need to identify a mechanism that erases the mass dependence as in the pressure hypothesis above.

Assuming that such a mechanism exists, what would this hypothesis predict for the global behavior? In this case $\left\langle r_{h}\right\rangle$ should be related to the mean density of the galaxy's dark matter halo, or

$$
\left\langle r_{h}\right\rangle \propto\left(M_{\text {vir }} / r_{\text {vir }}^{3}\right)^{-1 / 3}=\text { constant } \sim L_{\text {gal }}^{0}
$$

Therefore, this picture would also predict a roughly constant $\left\langle r_{h}\right\rangle$ across galaxies. It is interesting that both hypotheses predict roughly the observed global behavior. We conclude that the current observations cannot discriminate between these two scenarios. Indeed, the observational constraints seem to be ahead of what our 
limited knowledge of GC formation can predict, although it is clear that fundamental aspects of GC formation are encoded in them.

\section{HALF-LIGHT RADII AS A DISTANCE INDICATOR}

In the upper panel of Figure 9] we plot $\left\langle r_{h}\right\rangle$ in arcseconds versus inverse distance, $D$, for those galaxies in our sample with SBF distance measurements (Mei et al. 2005c). If $\left\langle r_{h}\right\rangle$ is constant in physical units, then we expect $\left(\left\langle r_{h}\right\rangle / 1^{\prime \prime}\right) \propto D^{-1}$ at low redshift (the dashed line). Although the vast majority of our sample galaxies are located at very nearly the same distance (i.e., the mean Virgo Cluster distance of $D=16.5 \pm 0.25 \mathrm{Mpc}$; Mei et al. 2005c), two galaxies in our sample give us some additional leverage to test this idea: (1) VCC 731 (NGC 4365) is a member of the Virgo W Cloud (de Vaucouleurs 1961) and has an SBF distance of $D=23.3 \pm 0.6$ Mpc (Mei et al. 2005c); and (2) NGC 4697, a nonmember of the Virgo Cluster which lies in the foreground, with an SBF distance of $11.3 \pm 0.5 \mathrm{Mpc}$ (Tonry et al. 2001; Mei et al. 2005c). ${ }^{18}$

The upper panel of Figure 9 reveals a scaling of $\left\langle r_{h}\right\rangle$ with $D^{-1}$ which has roughly the expected form, albeit with appreciable scatter for some of the fainter Virgo members. Note, however, that the half-light radii plotted in this panel have not been corrected for any dependence on GC or host galaxy properties as discussed in $\S 3$. In the bottom panel of this figure, we plot the average corrected half-light radii, $\left\langle\hat{r}_{h}\right\rangle$, against inverse distance. The dashed line, $\left\langle\hat{r}_{h}\right\rangle \propto D^{-1}$, is drawn so that $\left\langle\hat{r}_{h}\right\rangle$ has a value of 0 .'033 at our adopted mean distance of Virgo (Mei et al. 2005b). Note that line is not a fit, although it accurately predicts the measured $\left\langle\hat{r}_{h}\right\rangle$ for both VCC 731 and NGC 4697. It is clear that $\left\langle\hat{r}_{h}\right\rangle$ has the potential to be a powerful standard ruler for distance estimation. But as the upper panel of Figure 9] shows, even without any correction the directly measured $\left\langle r_{h}\right\rangle$ can be a useful distance indicator when restricted to the luminous galaxies (a class to which both VCC 731 and NGC 4697 belong). And trading some accuracy for simplicity, the mild nature of the required corrections means that a directly measured $\left\langle r_{h}\right\rangle$ can give a rough distance measurement for any early-type galaxy.

It should be noted that at least two other galaxies in the ACSVCS have celestial positions close to the W Cloud: VCC 571 and VCC 575. While they do not have enough GCs remaining after the selection process described in $\S 2$ to be included in our sample (they are left with just $3 \mathrm{GCs}$ each), their measured $\left\langle\hat{r}_{h}\right\rangle$ are nevertheless consistent with membership in the W Cloud. More specifically, VCC 571 has $\left\langle\hat{r}_{h}\right\rangle=0$ '. 023 , while VCC 575 has $\left\langle\hat{r}_{h}\right\rangle=0$.'021. An SBF distance is available only for VCC 575, which confirms that it is at a similar distance as VCC 731. While the number of GCs used to measure $\left\langle\hat{r}_{h}\right\rangle$ for VCC 575 is indeed low, it is reassuring that its measured value is consistent with it being at the distance of the $\mathrm{W}$ Cloud ( $\approx 23 \mathrm{Mpc}$ ), lending additional support to the validity of using $\left\langle\hat{r}_{h}\right\rangle$ as a distance indicator. The

\footnotetext{
18 Tonry et al. (2001) list the distance as $11.7 \mathrm{Mpc}$. To compare this value to our new SBF distance, the zeropoint must be adjusted to match that used in Mei et al. (2005b). As a consequence, the distance modulus in Tonry et al. (2001) should be shifted by -0.06 mag, so that $D=11.4 \pm 1.6 \mathrm{Mpc}$. This is in excellent agreement with our new measurement of $11.3 \mathrm{Mpc}$.
}

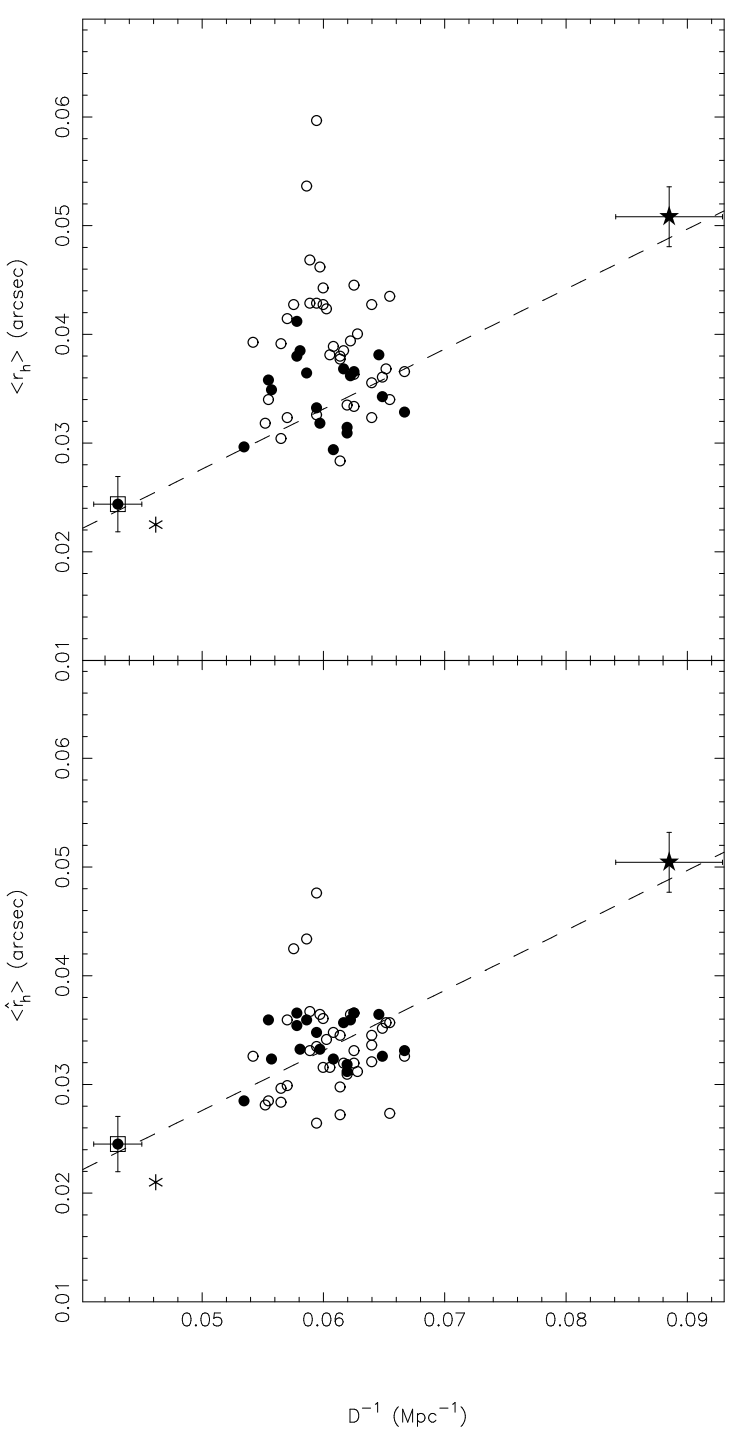

Fig. 9.- (Upper panel) $\left\langle r_{h}\right\rangle$ (no corrections) versus inverse distance $D^{-1}$. Filled symbols are galaxies with $M_{B}<-19$ mag, while open symbols are those with $M_{B} \geq-19$ mag. The star is NGC 4697 and the filled circle surrounded by a square is VCC 731. The dashed line represents the relation $\left\langle r_{h}\right\rangle \propto D^{-1}$ normalized to be $0 . \prime 033$ at $D=16.5 \mathrm{Mpc}$, and it is not a fit. Error bars are included only for NGC 4697 and VCC 731 for clarity. The asterisk is VCC 575. (Bottom panel) $\left\langle\hat{r}_{h}\right\rangle$ versus inverse distance $D^{-1}$. Filled symbols are galaxies with $M_{B}<-19$ mag, while open symbols are those with $M_{B} \geq-19$ mag. The star is NGC 4697 and the filled circle surrounded by a square is VCC 731 . The dashed line represents the relation $\left\langle r_{h}\right\rangle \propto D^{-1}$ normalized to be $0 . \prime 033$ at $D=16.5 \mathrm{Mpc}$, and it is not a fit. Error bars are included only for NGC 4697 and VCC 731 for clarity. The asterisk is VCC 575.

position in the sky and the measured $\left\langle\hat{r}_{h}\right\rangle$ for VCC 571 suggest that it is indeed a member of the $\mathrm{W}$ Cloud.

We now put forward the following method and calibration for the use of GC half-light radii in distance estimation. If a set of $\left\{r_{h, i}\right\}$ are measured on a given galaxy they should first be individually corrected for their color, underlying surface brightness and the host galaxy color by computing $\hat{r}_{h, i}$ according to equation [8] The surface brightness can be measured from the same frame where the GCs are detected and analyzed, and all of the quantities needed to perform the correction are distance independent. Then, if the median of the $\hat{r}_{h, i}$ is $\left\langle\hat{r}_{h}\right\rangle$ in 
arcseconds, the distance in Mpc follows from

$$
D=(0.552 \pm 0.058)\left(\frac{\left\langle\hat{r}_{h}\right\rangle}{1^{\prime \prime}}\right)^{-1} \mathrm{Mpc}
$$

where the uncertainty reflects the uncertainty in the assumed mean Virgo distance and the PSF systematics. While the expression above is in principle applicable to any early-type galaxy within the color/luminosity range spanned by our sample galaxies [i.e., $1.1 \lesssim(g-z)_{\text {gal }} \lesssim$ 1.6 and $\left.-22 \lesssim M_{B} \lesssim-15.5\right]$, in practice this technique would be applied almost exclusively to luminous giants which have populous GC systems, and where large samples of GCs can be easily accumulated and contamination is less of an issue. Additionally, in equation 8 measurements of the $(g-z)$ colors for the GC and galaxy are required, which would entail observing in these same bandpasses or transforming the color in hand to $(g-z)$, which would add some uncertainty. Needless to say, this also means that observations in at least two bands are needed to perform the correction. Thus, we note that for luminous galaxies (i.e., those with $M_{B} \sim-21 \mathrm{mag}$ ) a distance may be obtained without the benefit of color information by correcting the set of $r_{h, i}$ with the following approximation to equation 8

$$
\hat{r}_{h} \simeq r_{h} 10^{-0.016\left(\mu_{z}-21\right)+0.01} .
$$

The distance then follows by applying equation [19] The additional uncertainty incurred by this approximation if applied to a giant elliptical can be estimated to be of order a few percent. Of course $M_{B}$ won't be known beforehand and it would not be possible to know if the approximation is valid. In practice, this complication can be circumvented by applying the method only to the $\sim 10$ brightest early-type members of a given galaxy cluster.

What accuracy can be expected from this method? The rms deviation from a constant value $\left\langle r_{h}\right\rangle=0$ ". 0333 among our Virgo galaxies is 0.'0034 (where VCC 731 has been excluded). Thus, a single measurement can be expected to give a distance with external accuracy of $\approx 11 \%$. It is interesting to see that using our sample of $\left\langle\hat{r}_{h}\right\rangle$ we can already set an upper limit to the depth of Virgo (once again, excluding the background W Cloud). Assuming that $\left\langle\hat{r}_{h}\right\rangle$ is strictly constant and neglecting observational uncertainties, the observed dispersion translates into a distance dispersion of $\sigma_{D} \lesssim 1.7$ Mpc, or a $1-\sigma$ line-of-sight depth of $3.4 \mathrm{Mpc}$. While this finding is in good agreement with several previous measurements of the depth of Virgo around M87 (e.g., West \& Blakeslee 2000, Neilsen \& Tsvetanov 2000, Jerjen et al. 2004 ), it effectively rules out the claim of Young \& Currie (1995) that the Virgo Cluster has a very elongated distribution (extending from $\sim 8-20 \mathrm{Mpc}$ ) along the line of sight. Thus, this approach complements previous evidence against this claim from SBF measurements (Jerjen et al. 2004; Mei et al. 2005c).

\subsection{Geometrical Calibration and an Independent Distance to Virgo}

Up to now we have relied on our SBF distance measurements to establish the physical size of GCs in Virgo and to provide a calibration for the use of half-light radius as a distance indicator. Being reliant on SBF means that our distance measurement method is tied to the primary calibrators of the SBF method itself, and in particular to the calibration of the Cepheids. In this section, we calibrate the method using the Milky Way GC system only, thereby providing a simple geometric calibration of the method which is largely independent of any other extragalactic distance measurement method.

First, we note that all of the dependencies used above to compute corrected radii, $\hat{r}_{h, i}$, are independent of our SBF distances. Thus, we keep the form of these dependencies intact and attempt derive the zeropoint for the physical size using the Milky Way GC system. Of course, this approach faces has the difficulty of estimating the parameters needed for the corrections for the Milky Way, and the complication that our Galaxy is a disk system as opposed to the early-type galaxies targeted in the ACSVCS. These caveats notwithstanding, we now estimate a rough zeropoint to provide a direct calibration.

The quantities needed to determine $\left\langle\hat{r}_{h}\right\rangle$ for Galactic GCs are the $(g-z)$ colors of the GCs and the Galaxy itself, and the surface brightness profile in $z$. Instead of working with the latter, we choose to use $\log \left(r_{\mathrm{p}} / r_{e}\right)$, where for the effective radius we adopt the value given by de Vaucouleurs \& Pence (1978), $r_{e} \approx 5 \mathrm{kpc}$. We then use the Harris (1996) catalog of Galactic GCs to obtain the $r_{h},[\mathrm{Fe} / \mathrm{H}]$ and galactocentric distances $R_{\mathrm{gc}}$. In order to estimate their $(g-z)$ colors, which are not directly available, we transform from $[\mathrm{Fe} / \mathrm{H}]$ to $(g-z)$ as in Jordán et al. (2004b) (which assume an age of 13 Gyr). Finally, we take the integrated $(g-z)_{\text {gal }}$ color of our Galaxy to be $(g-z)_{\mathrm{Sbc}}=1.17$, the value appropriate for a type Sbc galaxy according to Fukugita et al. (1995). With these quantities in hand, we obtain a set of corrected half-light $\left\{\hat{r}_{h, i}\right\}$ radii using the prescriptions discussed above, namely

$\hat{r}_{h, i}=r_{h, i} 10^{\left.-0.07 \log \left(r_{\mathrm{p}} / 5\right)+0.17[(g-z)-1.2)+0.17(g-z)_{\mathrm{Sbc}}-1.5\right]}$,

for each of 100 simulated Galactic datasets with randomly projected galactocentric distance and with GCs selected according to our selection procedures (cf. $\S 3.2$ ).

From these simulations, we find $\left\langle\hat{r}_{h}\right\rangle \approx 2.54 \pm 0.1 \mathrm{pc}$ for the Galactic GC system. Note that the quoted uncertainty does not include systematics arising from our assumed properties of the Galaxy. These can be roughly estimated to be at least $15 \%$ by varying the assumed $r_{e}$ by $20 \%$ and changing the assumed color to that of a galaxy of type Sab and Scd. Using the fact that the observed $\left\langle\hat{r}_{h}\right\rangle$ for our sample is $0 . \prime 033$, we find that the mean distance of Virgo using this direct calibration is $16 \pm 2.3 \mathrm{Mpc}$. While this calibration should be viewed with some caution (being based on a late-type galaxy for which we do not have a direct measurement of its global characteristics), it is comforting to see that the distance of Virgo is in good agreement with other methods. Moreover, because this estimate circumvents several of the problematic issues involved in other methods used to establish Virgo's distance, such as the metallicity dependence of the Period-Luminosity relation for Cepheid variables and the distance modulus to the Large Magellanic Cloud, it provides a largely independent confirmation of the Cepheid/SBF distances.

\section{THE FORM OF THE $R_{H}$ DISTRIBUTION}


So far we have examined only the dependencies and properties of a location parameter of the distribution of GC half-light radii in early-type galaxies. In fact, in applying the various corrections to the set of $r_{h, i}$, we have tacitly assumed that only $\left\langle r_{h}\right\rangle$ was changing and that the shape of the $r_{h}$ distribution is invariant. We now show that this assumption is well justified and present an analytic form which accurately describes the observed $r_{h}$ distributions. Throughout this section, we will work with the fully corrected half-light radii, $\hat{r}_{h}$ and we additionally include galaxies that have less than 6 GCs after the cuts described in $\S 2$

To study the form of the distribution of half-light radii across the color and luminosity range of our galaxies, we chose to create bins in galaxy color. To minimize statistical noise, we require that each subsample contains at least 500 GCs (the reddest galaxy, VCC 1978, is assigned a bin of its own although it does not satisfy this condition). The resulting $\hat{r_{h}}$ distributions are shown in Figure 10] along with the distribution of the combined sample. Each panel shows a Gaussian kernel density estimate of the observed $\log \hat{r}_{h}$ distribution, with a bandwidth $h=0.035$ (solid curves). The corresponding $99 \%$ confidence bands are indicated by the dotted curves. It is apparent that the distribution of $\log r_{h}$ is very similar across the galaxy color range of our sample. The primary features are a prominent peak at $\hat{r_{h}} \sim 2.5 \mathrm{pc}$ and an extended, large- $\hat{r}_{h}$ tail which is much heavier than if the distribution was normal. The distribution is noticeably skewed, with an excess on the right side of the peak.

We emphasize that the distributions shown in Figure 10 are rather uncertain for $\hat{r}_{h} \lesssim 1 \mathrm{pc}$, as the measurements in that regime begin to suffer from biases to slightly larger $r_{h}$, due to the fact that the GCs are close to being unresolved. This difficulty notwithstanding, the decline toward small $r_{h}$ is certainly real. Unfortunately, the data do not allow us to characterize the regime $\hat{r}_{h} \lesssim 1$ pc accurately, so the following discussion should be taken to apply to the range $1 \lesssim \hat{r}_{h} \lesssim 10 \mathrm{pc}$ only. Note that the upper radius is imposed by our sample selection; in practice, this limit is set by the difficulty of distinguishing extended GCs from background galaxies. Thus, highly extended GCs, which certainly exist in our Galaxy, are not considered here. In any case, there appears to be no sudden upturn in the distribution for $\hat{r}_{h} \lesssim 1$ pc or $\hat{r}_{h} \gtrsim 10$ pc (see, e.g., Figure 12 of Haşegan et al. 2005), so it is clear that the bulk of the distribution lies in the regime accesible to us.

We have devised an analytical distribution function $\mathcal{J}$ with four parameters which is able to describe the observed distribution of $\log \hat{r}_{h}$, such that the probability of finding a GC with $\log \hat{r}_{h}$ in an interval of width $d\left(\log \hat{r}_{h}\right)$ around $\hat{r}_{h}$ is given by $\mathcal{J}\left(\log \hat{r}_{h}\right) d\left(\log \hat{r}_{h}\right)$. The form of this function is

$\mathcal{J}\left(\log \hat{r}_{h} \mid \Theta\right)=f \mathcal{G}\left(\log \hat{r}_{h} \mid \mu, \beta_{1}\right)+(1-f) \mathcal{G}\left(-\log \hat{r}_{h} \mid-\mu, \beta_{2}\right)$

where

$$
\begin{aligned}
& \mathcal{G}(x \mid \mu, \beta)=\frac{1}{\beta} \exp [-(x-\mu) / \beta-\exp (-(x-\mu) / \beta)], \\
& \Theta \equiv\left(\mu, \beta_{1}, \beta_{2}, f\right), \beta>0 \text { and } 0 \leq f \leq 1^{19}
\end{aligned}
$$

\footnotetext{
19 The function $\mathcal{G}$ can be derived as the distribution of extreme
}

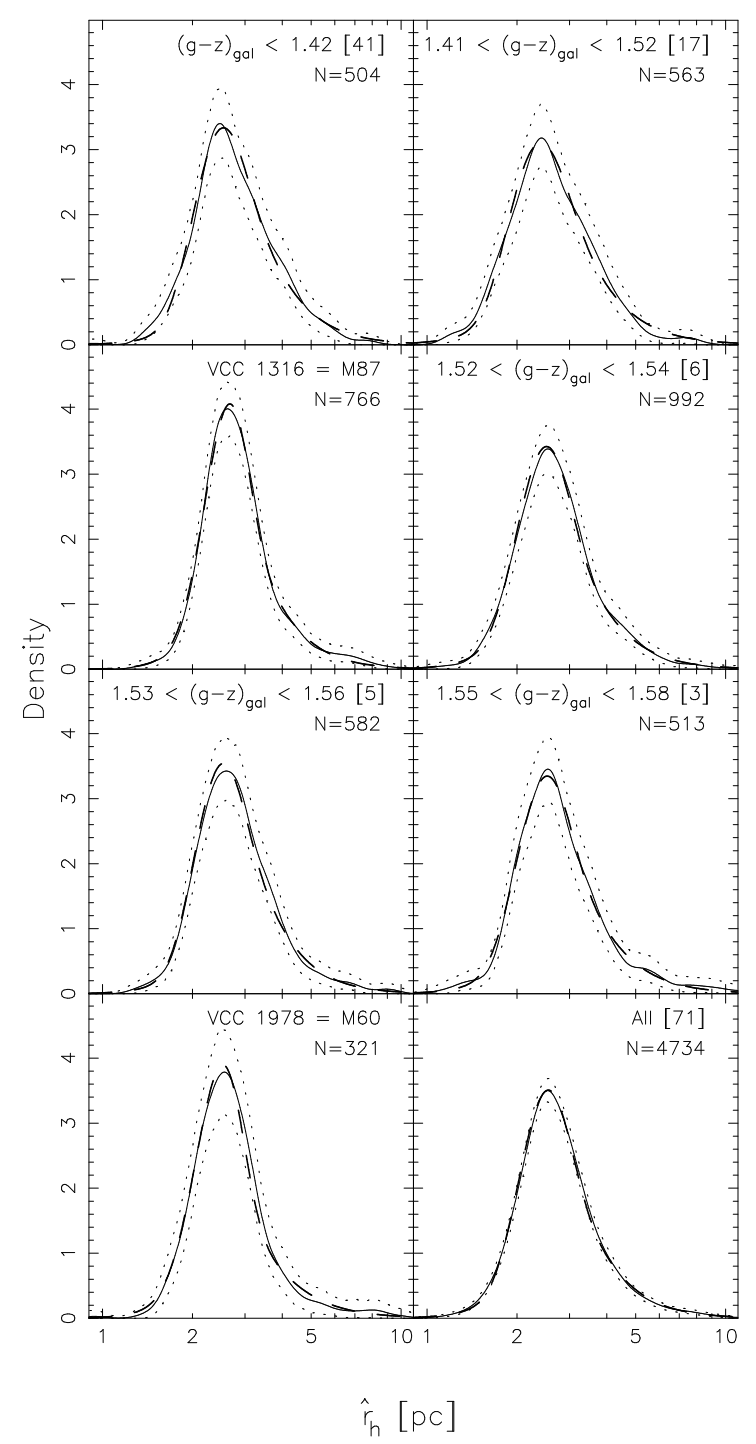

FIG. 10.- Distribution of $\log \hat{r}_{h}$ for GC samples built by combining galaxies in galaxy color bins as described in the text. The color bins (or the name of the individual galaxies if only one is present) are indicated in the different panels. The number of galaxies used in creating the combined GC sample is indicated in brackets and the number of GCs included is indicated by the value of $N$. The bottom right panel is built by combining all galaxies in the sample. The solid curves show Gaussian kernel density estimates with a bandwidth $h=0.035$; the dotted curves show their $99 \%$ confidence bands. The thick dashed line represents the best-fit function $\mathcal{J}$ (Equation 22) convolved with a Gaussian with $\sigma=h$.

The equivalent form of this distribution in terms of $\hat{r}_{h}$, such that the probability of finding a GC with $\hat{r}_{h}$ in an interval $d \hat{r}_{h}$ around $\hat{r}_{h}$ is given by $\tilde{\mathcal{J}}\left(\hat{r}_{h}\right) d \hat{r}_{h}$, is

$$
\tilde{\mathcal{J}}\left(\hat{r}_{h} \mid \tilde{\Theta}\right)=\tilde{f} \tilde{\mathcal{G}}\left(\hat{r}_{h} \mid \tilde{\mu}, \tilde{\beta}_{1}\right)+(1-\tilde{f}) \hat{r}_{h}^{-2} \tilde{\mathcal{G}}\left(\hat{r}_{h}^{-1} \mid \tilde{\mu}^{-1}, \tilde{\beta}_{2}\right)
$$

where

$$
\tilde{\mathcal{G}}(x \mid \tilde{\mu}, \tilde{\beta})=\frac{1}{x \tilde{\beta}}\left[\frac{x}{\tilde{\mu}}\right]^{-1 / \tilde{\beta}} \exp \left(-(x / \tilde{\mu})^{-1 / \tilde{\beta}}\right),
$$

values for independent identically distributed random variables. While this fact might hold some clue as to the physical basis for the observed distribution, we use it here strictly as a statistical modelling function without an a priori physical justification. 
$x, \tilde{\mu}, \tilde{\beta}>0,0 \leq \tilde{f} \leq 1$ and the parameters $\tilde{\Theta} \equiv$ $\left(\tilde{\mu}, \tilde{\beta}_{1}, \tilde{\beta}_{2}, \tilde{f}\right)$ are related to the ones in $\mathcal{J}$ by the relations $\tilde{\mu}=10^{\mu}, \tilde{\beta}_{1,2}=\ln (10) \beta_{1,2}$ and $\tilde{f}=f$. These functions satisfy $\int_{-\infty}^{\infty} \mathcal{J}\left(\log r_{h}\right) d\left(\log r_{h}\right)=\int_{0}^{\infty} \tilde{\mathcal{J}}\left(r_{h}\right) d r_{h}=1$.

We have obtained the best-fit parameters $\mu, \beta_{1}, \beta_{2}$ and $f$ via maximum-likelihood for the various subsamples shown in Figure 10 as well as for the combined sample of GCs. The resulting functions, convolved with a Gaussian with $\sigma=0.035$ in order to faithfully compare them with the density estimates, are shown in Figure 10 with a thick dashed curve. The function is able to produce a close match to the observed distributions.

The maximum-likelihood parameters obtained for each galaxy subsample are fairly consistent with the parameters inferred for the combined sample of all galaxies. This is true despite the fact that uncertainties arising from the effect of distance uncertainties in combining the $\hat{r_{h}}$ values for several galaxies are not included in the formal error estimates. The parameters for the combined sample, with uncertainties reflecting the dispersion of the values inferred for all galaxy color bins, are

$$
\begin{aligned}
& \mu=0.407 \pm 0.013 \\
& \beta_{1}=0.117 \pm 0.005 \\
& \beta_{2}=0.078 \pm 0.011 \\
& f=0.7 \pm 0.1
\end{aligned}
$$

and

$$
\begin{aligned}
\tilde{\mu} & =2.55 \pm 0.08 \\
\tilde{\beta}_{1} & =0.27 \pm 0.012 \\
\tilde{\beta}_{2} & =0.18 \pm 0.025 \\
\tilde{f} & =0.7 \pm 0.1
\end{aligned}
$$

We conclude that the observed distribution of $\hat{r}_{h}$ is well represented by single distributions of the form

$$
\mathcal{J}\left(\log \hat{r}_{h} \mid \mu=0.407, \beta_{1}=0.117, \beta_{2}=0.078, f=0.7\right)
$$

or, equivalently,

$$
\tilde{\mathcal{J}}\left(\hat{r}_{h} \mid \tilde{\mu}=2.55, \tilde{\beta}_{1}=0.27, \tilde{\beta}_{2}=0.18, \tilde{f}=0.7\right) .
$$

We now examine the applicability of the proposed function to the case of the Galactic GC system. In Figure 11 we show a Gaussian kernel density estimate with $h=0.06$ of the distribution of $\log \hat{r}_{h}$ for all GCs in our Galaxy satisfying $\hat{r}_{h}<10 \mathrm{pc}$ and $M_{V}<-7.4 \mathrm{mag}$, along with the $99 \%$ confidence bands. The individual $r_{h, i}$ have been corrected as described in $\S[$ We also show the bestfit form of $\mathcal{J}$ shown in equation 28 and convolved with a Gaussian with $\sigma=0.06$ as a dashed curve. Without any adjustment the form of the $\hat{r}_{h}$ distribution determined from our sample of early-type galaxies is able to reproduce satisfactorily the form observed in the Milky Way although the mean value is differs slightly. ${ }^{20}$ The dashdotted curve shows the same curve after setting $\mu=0.37$, and shows a very good agreement. This exercise supports the flexibility of the proposed form for describing the GC size distributions in galaxies of widely different mass and morphological type.

\footnotetext{
20 This mismatch would be reduced if we had used the distance inferred to Virgo from the direct geometric calibration using the Galactic GC system
}

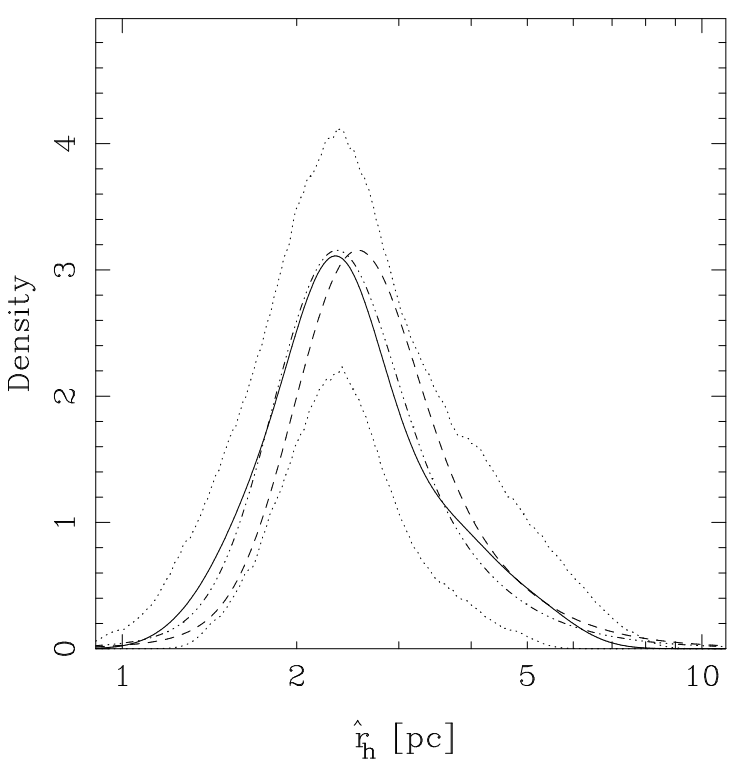

FIG. 11.- Distribution of $\log \hat{r}_{h}$ for Galactic GCs satisfying $M_{V} \leq-7.4 \mathrm{mag}$ and $0.75<r_{h}<10 \mathrm{pc}(66 \mathrm{GCs})$. The solid curve shows a Gaussian kernel density estimate with a bandwidth $h=$ 0.06 , while the dotted curves shows its $99 \%$ confidence bands. The dashed line represents the distribution given in equation [28] while the dash-dot-dot curve represents the same curve with $\mu=0.37$.

It is worth emphasizing that we have only modelled the observed distribution of $\hat{r}_{h}$, which is the convolution of the intrinsic distribution with the error distribution. If the measurement uncertainties are large compared to the width of the underlying distribution, then the observed distribution might be telling us more about the measurement errors than the actual distribution of $\hat{r_{h}}$. However, as noted in $\S 2$ the typical random uncertainties for our sample are of order $0^{\prime \prime} 003$ or $\approx 0.25 \mathrm{pc}$ at the mean distance of Virgo, with the systematic uncertainties estimated to be of the same order. The magnitude of the errors will thus affect the width of the observed peak to some extent, although the extended, high- $\hat{r_{h}}$ tail will be largely unaffected.

To investigate the effect of random uncertainties in a more quantitative way, we have taken the bestfit function of the form of equation [24 for VCC1316 $(\mathrm{M} 87=\mathrm{NGC} 4486)$ and determined the best match, via least squares, of a function with this same form but convolved with a Normal distribution with $\sigma=0.25 \mathrm{pc}$ in order to simulate the effect of the errors. The use of a single galaxy for this comparison ensures that we consider only random uncertainties: i.e., we are probing only the shape of the distribution, and not the absolute value of its location parameter. Figure 12 shows the result of this exercise. This figure demontrates that the inferred intrinsic distribution shares the same features as the one after convolution, suggesting that the observed distribution is indeed revealing intrinsic properties. Additionally, the fact that $\mathcal{J}$ also provides a close match to the Galactic data, which has a completely independent error distribution, further shows that the form of $\mathcal{J}$ traces the intrinsic properties of the $\hat{r_{h}}$ distribution.

Strictly speaking, the above analysis has relied on GCs that are brighter than the peak of the GC luminosity function, so our results apply in that regime only. While 


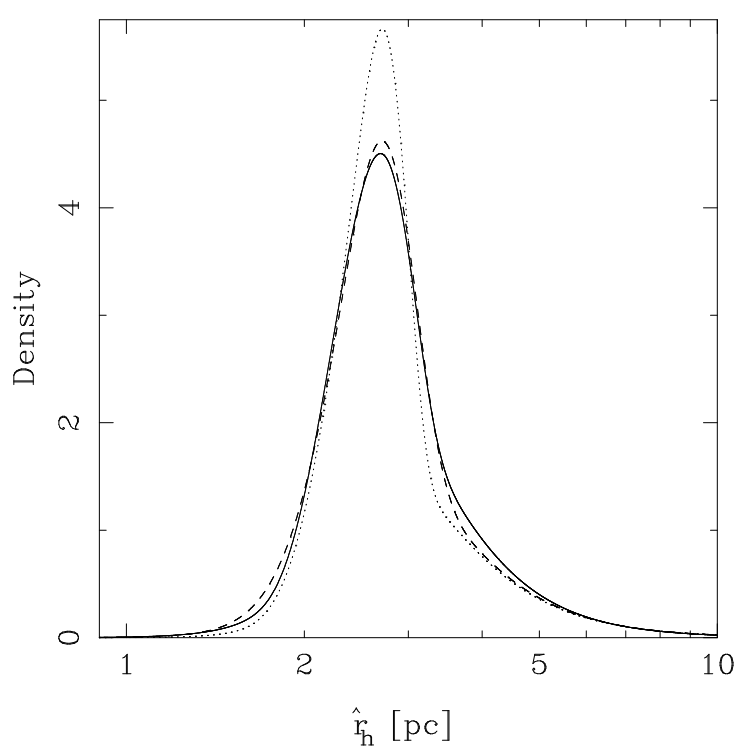

FIG. 12.- The solid line shows the best-fit function $\mathcal{J}$ to the $r_{h}$ distribution of VCC1316 $(\mathrm{M} 87=$ NGC 4486). The dotted line shows a function of the same form which best matches the observed data for M87 after convolution with the average random uncertainty in the $r_{h}$ measurement (assumed to be a Normal distribution with $\sigma=0.25 \mathrm{pc}$ ). The convolved function is shown as the dashed line.

it is reasonable to expect the distribution to apply to the less luminous GCs, this claim needs to be tested. Indeed, if we considered all Galactic GCs in Figure [1] then the agreement would be somewhat worsened due to the presence of a larger proportion of faint, extended clusters. While the form of the distribution is still well described by $\mathcal{J}$, the shape and location parameters need to be changed somewhat from those presented in equation 28. In particular, the extended high- $\hat{r}_{h}$-tail becomes more important when the fainter GCs are included in the analysis, which is reflected by the larger $\beta_{1}$ (i.e., 0.16 , as opposed to the value of 0.12 obtained when only the bright GCs are considered).

The form of the distribution presented above should serve are a useful constraint for models of GC formation and numerical simulations of their dynamical evolution. Any viable picture of star formation in clusters should produce an observed size distribution that is consistent with the form of $\mathcal{J}$, at least for GCs brighter than the luminosity function turnover. The fact that its form seems to be uniform across the wide range in luminosity and color spanned by ACSVCS program galaxies strongly suggests that some quite generic mechanism determines $\mathcal{J}$, while the location of the distribution is set by the local properties of the GC system and/or its host galaxy.

\section{SUMMARY \& CONCLUSIONS}

We have analysed the half-light radii of GCs in earlytype galaxies using the exceptional dataset provided by the ACS Virgo Cluster Survey. We have presented evidence that the average half-light radii $\left\langle r_{h}\right\rangle$ of GCs belonging to early-type galaxies increases with galactocentric distance or, alternatively, with decreasing surface brightness. This dependence is significantly shallower than that observed in our Galaxy. For the first time, we report an increase in $\left\langle r_{h}\right\rangle$ with decreasing galaxy color $(\sim$ decreasing luminosity), albeit with modest statistical significance. In agreement with previous observations, we find strong evidence that $\left\langle r_{h}\right\rangle$ is independent of the luminosity (mass) of GCs. We also find $\left\langle r_{h}\right\rangle$ to be $\sim 17 \%$ smaller for red (metal-rich) than for their blue (metalpoor) counterparts. This trend is better explained by a mechanism that is intrinsic to GCs rather than to projection effects. As such, the observations favor the picture in which this difference follows from the combined effects of mass segregation and the metallicity dependence of stellar lifetimes under the assumption of invariant average half-mass radii (Jordán 2004).

We have discussed the predictions of two simple pictures for the origin of the $r_{h}$ of GCs: one based in the idea that they are determined by the average ambient pressure surrounding proto-GC clouds, and other that $r_{h}$ is related to the tidal radius set by the gravitational field of the host galaxy. Both pictures are roughly consistent with the observed behavior, $\left\langle r_{h}\right\rangle \sim L^{0}$, although some elements of the theoretical framework are lacking in both scenarios. While our level of understanding of GC formation is still in its infancy, it is clear that the observations of the $r_{h}$ of GCs can provide important insights into the physical mechanisms which have shaped their formation and evolution.

We have studied the form of the observed distribution of GC half-light radii in early-type galaxies. Once corrected for a variety of dependencies on color, radius and surface brightness, the form of this distribution is remarkably consistent across the range of color and luminosity spanned by our sample of galaxies. We introduce simple analytic expressions (equations 22 and 24) which satisfactorily describe the form of the $\hat{r}_{h}$ distribution in our sample galaxies and in the Milky Way. Future theories of GC formation and evolution should be able to produce results consistent with this form when predicting the observed distribution of $r_{h}$.

Once corrected for dependencies on color and surface brightness, the mean half-light radii of GCs can be used as a standard ruler for distance estimation. Specifially, we find a constant value of $\left\langle\hat{r_{h}}\right\rangle=2.7 \pm 0.3 \mathrm{pc}$ for a GC with color $(g-z)=1.2$, in a galaxy with color $(g-z)_{\text {gal }}=1.5$, and at an underlying $z$-band surface brightness of $\mu_{z}=21 \mathrm{mag} \operatorname{arcsec}^{-2}$. A first attempt at a geometric calibration of this method based on the Galactic GC system gives an independent distance to the Virgo Cluster of $16 \pm 2.3 \mathrm{Mpc}$. While these conclusions rest heavily on two datapoints besides the bulk of galaxies in Virgo (VCC 731 and NGC 4697), the ongoing ACS Fornax Cluster Survey (Jordán et al., in preparation), which is similar to the ACSVCS but focusing on 43 earlytype members of the Fornax Cluster, will be invaluable in this regard by adding many additional datapoints at $D \sim 19$ Mpc (Tonry et al. 2001).

While not as efficient some other distance indicators, such as SBF, the use of $\left\langle\hat{r}_{h}\right\rangle$ as a distance indicator could, in principle, offer some advantages that could render it a valuable complement to other GC-based method such as the GC luminosity function. For instance, half-light radii are expected to remain roughly constant during the evolution of a GC. This is certainly not the case for luminosity, which will be affected by age and metallicity, 
quantities which are not necessarily known a priori. Additionally, it is not necessary to go past the turnover of the luminosity function if enough clusters to accurately determine $\left\langle\hat{r_{h}}\right\rangle$ can be accumulated up to brighter magnitudes. Assuming one could measure $\left\langle\hat{r}_{h}\right\rangle$ up to $1 / 4$ of the PSF FWHM of a diffraction-limited telescope of diameter $d$ at a wavelength of $1 \mu$, this technique could be applied out to distances of $\sim 50 \mathrm{Mpc}(d=6 \mathrm{~m}), \sim 250$ $\operatorname{Mpc}(d=30 \mathrm{~m})$ and $\sim 850 \mathrm{Mpc}(d=100 \mathrm{~m})$, illustrating the prospect for its implementation with future observing facilities. In practice, the limitation will be most likely set by the difficulty of accurately characterizing the PSF over a sufficiently large field of view.

P.C. would like to thank the European Southern Observatory and especially Bruno Leibundgut for their hos- pitality during the preparation of this paper. Support for programs GO-9401 and GO-10003 was provided through grants from the Space Telescope Science Institute, which is operated by the Association of Universities for Research in Astronomy, Inc., under NASA contract NAS526555. P.C. acknowledges additional support provided by NASA LTSA grant NAG5-11714. M.M. acknowledges additional financial support provided by the Sherman M. Fairchild foundation. D.M. is supported by NSF grant AST-020631, NASA grant NAG5-9046, and grant HSTAR-09519.01-A from STScI. M.J.W. acknowledges support through NSF grant AST-0205960. This research has made use of the NASA/IPAC Extragalactic Database (NED) which is operated by the Jet Propulsion Laboratory, California Institute of Technology.

\section{APPENDIX}

\section{KINGPHOT: AN ALGORITHM FOR THE MEASUREMENT OF GC PHOTOMETRIC AND STRUCTURAL PARAMETERS USING HST/ACS IMAGES}

Measurements of the photometric and structural parameters of GCs in the ACSVCS, including their half-light radii, were carried out with code specifically written for these purposes. This code, hereafter referred to as KINGPHOT, was designed to perform efficient and accurate $r_{h}$ measurements for the thousands of GCs detected in the ACS Virgo Cluster Survey. The primary technical challenge in the design of the code is to recover $r_{h}$ for the case of marginally resolved objects: i.e., objects with intrinsic sizes comparable to, or smaller than, the instrumental PSF. In this appendix, we outline the methodology used by the code and present some simulations that illustrate its performance.

What is observed at the detector is an integral over each pixel of the PSF-convolved object; note that we include in the term PSF the effects of the telescope optics and detectors. The exact nature of the physical effects that give rise to the observed light distribution are of no concern to us here, as long as we have an empirical way of determining the final distribution on the detector of the light from a celestial point source. With this definition of the PSF, $\mathcal{P}$, the observed flux $O$ at a given pixel in the detector is given by

$$
O=\iint_{\text {pixel }} d x_{1} d x_{2}[\mathcal{P} \otimes \mathcal{F}]\left(x_{1}, x_{2}\right)
$$

where $\mathcal{F}$ is the flux distribution of the object and $\otimes$ denotes a convolution.

If $\mathcal{F}$ can be described parametrically by a set of parameters $\Theta$, and if a model for $\mathcal{P}$ is available, then the parameters in $\boldsymbol{\Theta}$ can be simply estimated by $\chi^{2}$ minimization of the observed two-dimensional light distribution minus a predicted model. Because the family of steady-state dynamical models presented by King (1966) are known to provide an excellent match to the observed light profiles of most Galactic GCs, we have chosen to parameterize $\mathcal{F}$ by these models. King models are a one-parameter family indexed by the central potential, $W_{0}$, or equivalently, by the concentration, $c \equiv \log _{10}\left(r_{t} / r_{c}\right)$, where $r_{t}$ is the tidal radius (the point at which the density is zero) and $r_{c}$ is the core radius (the point at which the density falls to roughly half of its central value). In addition to $c$, there are two arbitrary scaling factors: the total magnitude, $m$, and one related to spatial scale. The latter is typically taken to the core radius, but we choose to work with the half-light radius $r_{h}$. For King models, the ratio $r_{h} / r_{c}$ is uniquely determined by the concentration, so the choice of either is equivalent.

There is no analytic expression for the spatial density corresponding to the lowered Maxwellian phase space distribution assumed by King (1966), but the density is easily obtained by an integration of Poisson's equation. It would be prohibitively slow to compute this density profile by solving Poisson's equation during the $\chi^{2}$ minimization, as this would entail the computation of hundreds of models for each of the $\sim 10^{4} \mathrm{GC}$ candidates in the ACSVCS. Instead, a library of King-model density profiles was constructed, computed in a grid of 300 equally spaced points in $W_{0}$ ranging from $W_{0}=1$ to $W_{0}=12$, which corresponds to the range $0.296<c<2.739$. This library is read into memory at the start of KINGPHOT and a model of a given $c$ is obtained by spline interpolation between the two closest models in the library.

To represent $\mathcal{P}$, we adopt the method implemented in the DAOPHOT II program (Stetson 1987; 1993). We refer the reader to Stetson (1987) for the details of the PSF representation adopted. The PSFs for the current work were determined empirically using moderately crowded fields in the Galactic GC 47 Tucanae as described in Jordán et al. (2004a).

Given the adopted King model, the corresponding model parameters, and the PSF model, the predicted light distribution at the detector can be obtained by calculating the convolution in equation A1 The convolution is performed via a fast Fourier transform using routines from the Fastest Fourier Transform in the West library (FFTW; Frigo \& Johnson 2005)

Let us denote the observed data at a pixel $(i, j)$ by $d_{i j}$, and the prediction of a PSF-convolved King model centered at 
position $\left(x_{0}, y_{0}\right)$ in the detector by $O_{i j}\left(c, m, r_{h}, x_{0}, y_{0}\right)$. The parameters $\left(c, m, r_{h}, x_{0}, y_{0}\right)$ are estimated by minimizing the value of $\chi^{2}$ given by

$$
\chi^{2}=\sum_{\left\{|(i, j)|<r_{\mathrm{fit}}\right\}}\left[w_{i j}\left(d_{i j}-O_{i j}\left(c, m, r_{h}, x_{0}, y_{0}\right)\right) / \sigma_{i j}\right]^{2}
$$

where $\sigma_{i j}$ is the uncertainty of $d_{i j}$ and $w_{i j}$ are radial weights. The value of $\sigma_{i j}$ used by KINGPHOT is the expected $r m s$ in the data image given by equation (2) of Jordán et al. (2004a). In the above summation, $\left\{|(i, j)|<r_{\text {fit }}\right\}$ denotes all pixels within a distance $r_{\text {fit }}$ of $\left(x_{0}, y_{0}\right)$. The weights $w_{i j}$ are set to the value of the function $5 /\left(5+1 /\left(r_{\text {fit }}^{2} / r_{i j}^{2}-1\right)\right)$, where $r_{i j}$ is the distance of the pixel's center to $\left(x_{0}, y_{0}\right)$. These radial weights are included to assist convergence as discussed in Stetson (1987). Initially, the parameters for all GCs were determined using $r_{\text {fit }}=4$ pixels. Our simulations show that for extended clusters, with $r_{h} \gtrsim r_{\text {rfit }} / 2$, the measured $r_{h}$ starts to suffer from biases; for larger GCs, we increased the value of $r_{\text {rfit }}$ and redetermined the best-fit parameters. This was repeated until the condition $r_{h}<r_{\text {rfit }} / 2$ is met or $r_{\mathrm{fit}}=15$ pixel. This condition is satisfied for all GCs considered in this analysis.

Minimizations were carried with CERN's Minuit package. The minimization was done in two steps. First, a Simplex (Nelder \& Mead 1965) minimization algorithm was used, followed by a variable metric method with inexact line search (MIGRAD). In the Simplex minimization the concentration is held fixed at $c=1.5$, a typical value for Galactic GCs (Harris 1996). The starting values for the parameters $\left(m, x_{0}, y_{0}\right)$ are obtained from SExtractor (Bertin \& Arnouts 1996) while $r_{h}$ is set to $0^{\prime \prime} 0385$, roughly the angle subtended by $3 \mathrm{pc}$ at the Virgo distance. After the Simplex minimization, the center $\left(x_{0}, y_{0}\right)$ is fixed while $c$ is allowed to vary. Before starting the MIGRAD minimization, a grid search is done in $\left(c, r_{h}\right)$ and the minimization is started at the parameters that give the lowest $\chi^{2}$ in this grid search. This procedure is done to avoid biasing the final result by the choice of fixing $c$ in the Simplex minimization and to avoid converging to a local, rather than a global, minimum. After MIGRAD is run, the best fit parameters are recorded. To estimate the uncertainties a bootstrap procedure is used. From the oberved data $d_{i j}$ within the fitting radius, a random set of $d_{i j}$ is generated and the minimization is re-run, 50 times, with this new set. The output parameters recorded, and the $r m s$ of the recovered parameters is taken to represent the uncertainties.

To test the performance of this code, it has been run on a set of simulated GCs to which an appropriate amount of instrumental and Poisson noise has been added. For all combinations of $\left(c, r_{h}\right)$ in the grid $c=0.5,0.7, \ldots, 2.1,2.3$ and $r_{h}=0.5,1,2,4,8,16 \mathrm{pc}$ (at the distance of Virgo), 15 objects were simulated and the best-ft parameters recorded. This was done for instrumental magnitudes of $m=20,21,22,23,24,25$ (for a 1s exposure), with a fitting radius of $r_{\text {fit }}=4 \mathrm{WFC}$ pixels. The relevant results of these simulations are summarized in Figure A13 where we show the average $\Delta r_{h} / r_{h}$ (for all simulated $c$ values). Here, $\Delta r_{h}$ is the difference between the recovered and input $r_{h}$, Two sets of error bars are shown for each point, slightly displaced. The one to the left shows the observed $r m s$ of $\Delta r_{h} / r_{h}$ while the one to the right indicates the average uncertainty of $\Delta r_{h} / r_{h}$ returned by KINGPHOT. Instrumental magnitudes can be transformed into $z$-band ones by $m_{z} \simeq m-0.14$. Thus, only the four uppermost panels of Figure A13 are relevant to the present work (due to the construction of the sample; see Equation 11).

We conclude on the basis of these simulations that the performance of KINGPHOT is, for observational material similar to the ACSVCS, largely unbiased for $r_{h} \gtrsim 1 \mathrm{pc}$. At the same time, it is apparent that, for $r_{h} \lesssim 1 \mathrm{pc}$, there are always systematic biases which are of order $\lesssim 20 \%$. It is also apparent is that a bias arises when the intrinsic $r_{h}$ approaches $r_{\text {fit }}$, as is revealed by the rightmost point in each panel. These bias, however, is avoided if $r_{h} \lesssim 0.5 r_{\text {fit }}$, so it is necessary that this condition is satisfied post-facto by iteratively adjusting $r_{\text {fit }}$ as we have done (see above). Finally, the uncertainties returned by KINGPHOT agree very well with the observed dispersion on the $\Delta r_{h} / r_{h}$ values, and thus we conclude that our bootstrap procedure estimates the uncertainties properly. Needless to say, such simulations can provide information only on the intrinsic performance of the code, and neglect sources of error arising from inaccuracies in the assumed forms for $\mathcal{F}$ or $\mathcal{P}$. Uncertainties arising from systematic errors of this sort can be estimated from the independent measurements in the two bandpasses (see $\S 2$ ). 


\section{REFERENCES}

Aarseth, S.J., \& Heggie, D.C. 1998, MNRAS, 297, 794

Ashman, K.M., \& Zepf, S.E. 2001, AJ, 122, 1888

Barmby, P., Holland, S.T., Huchra, J.P. 2002, AJ, 123, 1937

Baum, W.A. 1959, PASP, 71, 106

Beers, T.C., Flynn, K., \& Gebhardt, K. 1990, AJ, 100, 32

Bertin, E. \& Arnouts, S. 1996, A\&AS, 117, 393

Binggeli, B., Sandage, A., \& Tammann, G.A., 1985, AJ, 90, 1681

Binney, J., \& Tremaine, S. 1987, Galactic Dynamics (Princeton: Princeton Univ. Press)

Bullock, J.S. et al. 2001, MNRAS. 321. 559

Cappellari, M., et al. 2005, astro-ph/0505042

Caon, N., Capaccioli, M., \& DU Unotrio, N1. 1993, MNRAS, 265, 1013

Côté, P., et al. 2001, ApJ, 559, 828

Côté, P., et al. 2004, ApJS, 153, 223

de Vaucouleurs, G. 1961, ApJS, 6, 213

de Vaucouleurs, G., \& Pence, W.D. 1978, AJ, 83, 1163

Dirsch, B., Richtler, T., Geisler, D., Forte, J.C., Bassino, L.P., \& Gieren, W.P. 2003, AJ, 125, 1908

Ferrarese, L. 2002, ApJ, 578, 90

Fukugita, M., Shimasaku, K., \& Ichikawa, T. 1995, PASP, 107, 945

Frigo, M., \& Johnson, S.G. 2005, Procceedings of the IEEE, 93(2), 216

Gunn, J.E., \& Griffin, R.F. 1979, AJ, 84, 752

Haşegan, M., et al. 2005, ApJ, 627, 203

Harris, W.E. 1996, AJ, 112, 1487

Jerjen, H., Binggeli, B., \& Barazza, F.D. 2004, AJ, 127, 771

Jordán, A. 2004, ApJ, 613, L117

Jordán, A. et al. 2004a, ApJS, 154, 509

Jordán, A. et al. 2004b, ApJ, 613, 279

King, I.R. 1966, AJ, 71, 64

Kissler-Patig, M., Richtler, T., Storm, J., della Valle, M. 1997, A\&A, 327, 503

Kundu, A., \& Whitmore, B.C. 1998, AJ, 116, 2841

Kundu, A., Whitmore, B.C., Sparks, W.B., Macchetto, F.D., Zepf, S.E., \& Ashman, K.M. 1999, ApJ, 513, 733
Kundu, A., \& Whitmore, B.C. 2001, AJ, 121, 2950

Larsen, S.S., Brodie, J.P., Huchra, J.P., Forbes, D.A., Grillmair, C. 2001, AJ, 121,2974

Larsen, S.S., Forbes, D.A., \& Brodie, J.P., 2001, MNRAS, 327, 1116

Larsen, S.S. \& Brodie, J. 2003, ApJ, 593, 340

Lee, M.G., Kim, E., \& Geisler, D. 1998, AJ, 115, 947

Lightman, A.P., \& Shapiro, S.L. 1978, Rev. Mod. Phys., 50, 437

McLaughlin, D.E., \& Pudritz, R.E. 1996, ApJ, 469, 194

McLaughlin, D.E. 2000, ApJ, 539, 618

Mei, S., et al. 2005a, ApJS, 156, 113

Mei, S., et al. 2005b, ApJ, 625, 121.

Murphy, B.W., Cohn, H.N., \& Hut, P. 1990, MNRAS, 245, 355

Murray, S.D., \& Lin, D.N.C. 1992, ApJ, 400, 265

Navarro, J.F., Frenk, C.S., \& White, S.D.M. 1995, MNRAS, 275, 720

Neilsen, E.H., Jr., \& Tsvetanov, Z.I. 2000, ApJ, 536, 255

Nelder, J. A., \& Mead, R. 1965, Comput. J., 7, 308

Peng, E.W. et al. 2005, submitted to ApJ

Puzia, T.H., Kissler-Patig, M., Brodie, J.P., \& Huchra, J.P. 1999, AJ, 118,2734

Shapley, H., \& Sawyer, H. 1927, Harv. Obs., 852, 22

Spitzer, L., \& Thuan, T.X. 1972, ApJ, 175, 31

Tonry, J.L., \& Schneider, D.P. 1988, AJ, 96, 807

Tonry, J.L., Dressler, A., Blakeslee, J.P., Ajhar, E.A., Fletcher, A.B., Luppino, G.A., Metzger, M.R., \& Moore, C.B. 2001, ApJ, 546,681

Tremonti, C.A., et al. 2004, Ap.J, 613, 898

van den Bergh, S. 1956, ZAp, 41, 61

van den Bergh, S. 1975, ARA\&A, 13, 217

van den Bergh, S., Morbey, C., \& Pazder, J. 1991, ApJ, 375, 594

West, M.J., \& Blakeslee, J.P. 2000, ApJ, 543, L27

Young, C.K., \& Currie, M.J. 1995, MNRAS, 273, 1141 
JORDÁN ET AL.

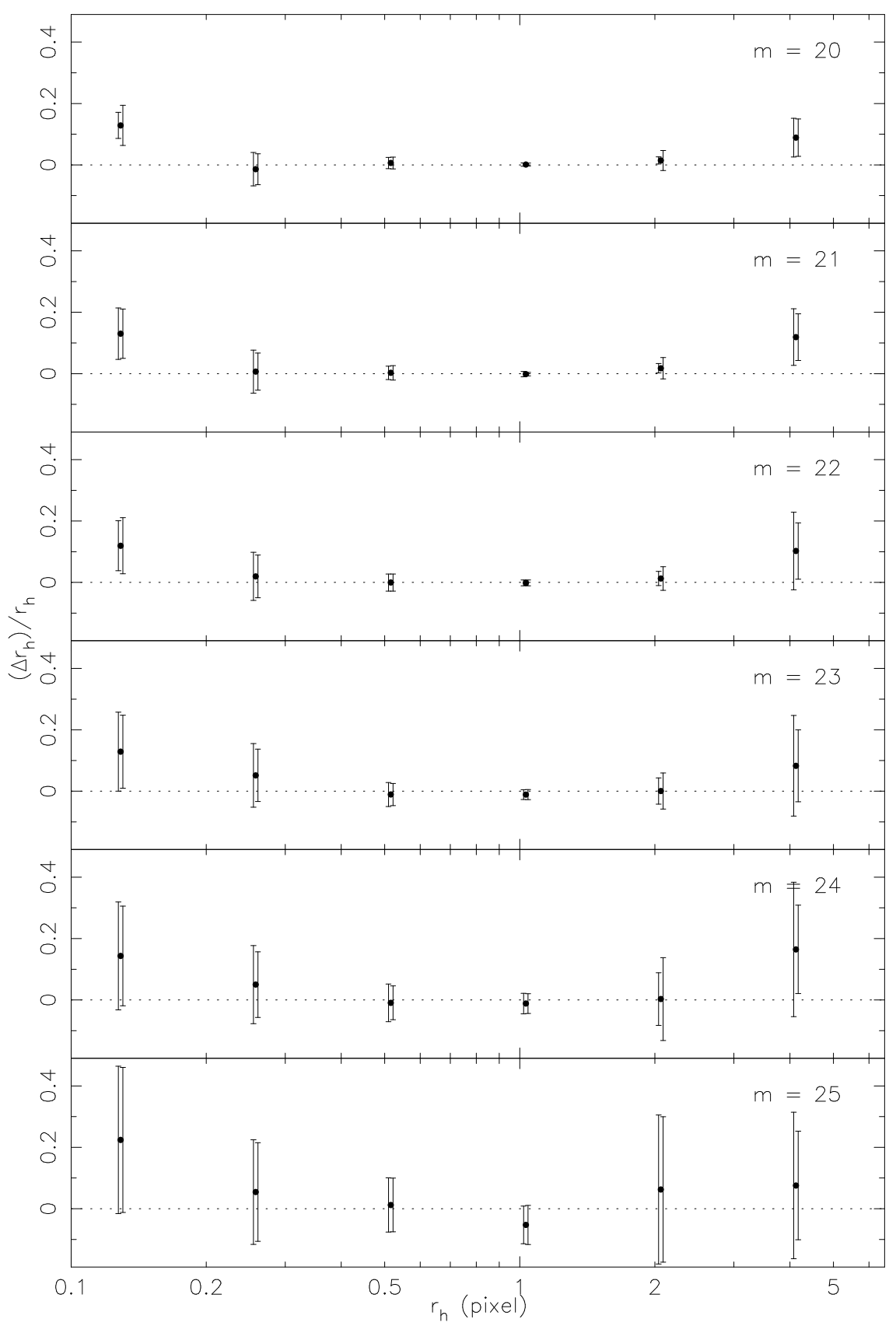

FIG. A13. $-\Delta r_{h} / r_{h}$ versus $r_{h}$ for a set of KINGPHOT runs on simulated GCs. The instrumental magnitude $m$ (for a 1 s exposure) of the simulated GCs is indicated in each panel. For each simulated $r_{h}$, we plot the fractional mean difference between the measured and input $r_{h}, \Delta r_{h} / r_{h}$. We also show two sets of error bars for each point: the left one is the observed rms of $\Delta r_{h} / r_{h}$ while the right one is the average uncertainty computed by the code. The fitting radius was set to $r_{\text {fit }}=4$ pixels. 